\title{
DỨBin
}

Technological University Dublin ARROW@TU Dublin

\section{Free Energies For Singleton Minimal States}

John Murrough Golden

Technological University Dublin, Murrough.Golden@TUDublin.ie

Follow this and additional works at: https://arrow.tudublin.ie/scschmatart

Part of the Mathematics Commons

\section{Recommended Citation}

Golden, M. (2016) Free energies for singleton minimal states. Continuum mechanics and thermodynamics, June 2016. Springer. doi:10.1007/s00161-016-0512-3

This Article is brought to you for free and open access by the School of Mathematics at ARROW@TU Dublin. It has been accepted for inclusion in Articles by an authorized administrator of ARROW@TU Dublin. For more information, please contact arrow.admin@tudublin.ie, aisling.coyne@tudublin.ie,gerard.connolly@tudublin.ie.

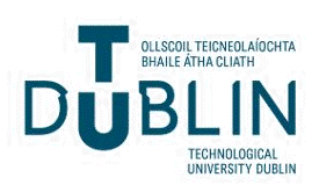




\title{
- Free energies for singleton minimal states
}

\author{
Received: 4 February 2016 / Accepted: 4 June 2016 \\ ○ Springer-Verlag Berlin Heidelberg 2016
}

\begin{abstract}
It is assumed that any free energy function exhibits strict periodic behavior for histories that have been periodic for all past times. This is not the case for the work function, which, however, has the usual defining properties of a free energy. Forms given in fairly recent years for the minimum and related free energies of linear materials with memory have this property. Materials for which the minimal states are all singletons are those for which at least some of the singularities of the Fourier transform of the relaxation function are not isolated. For such materials, the maximum free energy is the work function, and free energies intermediate between the minimum free energy and the work function should be given by a linear relation involving these two quantities. All such functionals, except the minimum free energy, therefore do not have strict periodic behavior for periodic histories, which contradicts our assumption. A way out of the difficulty is explored which involves approximating the relaxation function by a form for which the minimal states arc no longer singletons. A representation can then be given of an arbitrary free energy as a linear combination of the minimum, maximum and intermediate free energies derived in earlier work. This representation obeys our periodicity assumption. Numerical data are presented, supporting the consistency of this approach.
\end{abstract}

\section{Introduction}

There are generally many fiee energies relating to a given material with memory. They form a bounded convex set with a minimum and a maximum element ([11] and earlier references therein), which we will denote by $\mathcal{F}$.

Over the last 15 years, explicit algebraic representations of the minimum and related free energies have been given for materials with memory which have constitutive equations for stress ${ }^{1}$ given by linear functionals of the strain; see for example $[1,2,8,10,12-18]$.

More basic examples of free energies, in terms of the relaxation function, were constructed and considered in earlier references, notably the Graffi-Volterra and Dill functionals discussed in $[1,6,7,11,21,23]$ and other papers. There is also the Fabrizio free energy functional introduced in [9], which is an explicit functional of the minimal state (FMS).

${ }^{1}$ We consider for definiteness isothermal mechanical problems, indeed those for solid viscoelastic materials. Also, only the scalar case is considered, which simplifies the algebra and allows us to focus on the essential structure of the arguments. It must be emphasized, however, that similar results can be given with little extra difficulty, for viscoelastic fluids, some non-isothermal problems, electromagnetism, non-simple materials etc., and indeed for the general tensor cases relating to all these materials; the references noted above provide many such examples.

Communicated by Andreas Öchsner.

J. M. Golden (D)

School of Mathematical Sciences, Dublin Institute of Technology, Kevin Street, Dublin 8, Ireland

E-mail: murrough.golden@dit.ie

$$
\text { "161_2016_512_Article" — 2016/6/15 - 9:11 - page } 1 \text { - \#1 }
$$


These are all quadratic functionals which yield linear constitutive equations for the stress, in a mechanics context. A generalization of such quadratic functionals, yielding non-linear constitutive relations has been proposed recently [19].

One of the free energies in $\mathcal{F}$ is the physical free energy, the associated rate of dissipation which yields the rate of heat loss resulting from deformation. The problem of identifying this particular free energy is discussed in $[15,16]$, but more definitively addressed in $[20]$.

Some functionals, in particular those of Graffi-Volterra, Dill and Fabrizio, are free energies only for certain types of material, so that the size of $\mathcal{F}$ will depend on material properties. Those with the least constraints on the material are the minimum free energy and also the family of related functionals discussed in for example $[1,15]$ (though this family is non-trivial only for relaxation functions with no branch cut singularities in the frequency domain).

Recent work [17] seeks to provide methods for systematically exploring existing and new categories of free energy functionals in $\mathcal{F}$, based on a novel technique for constructing free energies. Quadratic functionals of quantities known to be FMSs are explored in [18].

If a free energy functional is to correspond in some sense to a measure of (finite) stored energy, it should exhibit strict periodic behavior for histories that have been periodic for all past times. This is not the case for the work function, which changes by a fixed amount over each cycle, although it has the usual defining properties of a free energy, with associated zero dissipation rate.

For materials where the minimal states are all singletons, the maximum free energy is the work function, and free energies intermediate between the minimum free energy and the work function are given by a linear relation involving these two quantities. All such functionals, except the minimum free energy, therefore share this property of the work functional that they change over each cycle. This difficulty is discussed in the present work, and a solution suggested.

Many of the developments of Sects. 2, 3, 4, 5, 6, 7,9, and 11 are well covered in earlier references, in particular [1], allowing us to omit most denvations. These developments provide the basis on which the new results, mainly concentrated in Sects. 8, 10, 12 and 13, are constructed.

Regarding the notational convention for referring to equations, we adopt the following rule. A group of relations with a single equation number (***) will be individually labeled by counting " $=$ " signs or " $<$ ", " $>$ ", " $\geq$ " and " $\leq$ ". Thus, $(* * *)_{5}$ refers to the fifth " $=$ "sign, if all the relations are equalities. Relations with " $\in$ " are ignored for this purpose.

\section{Basic relations}

The strain at time $t$ and its history are $E(t)$ and $E^{t}$, where

$$
E^{\dagger}(s)=E(t-s), \quad s \in \mathbb{R}^{+},
$$

while the relative history is given by

$$
E_{r}^{t}(s)=E^{t}(s)-E(t), \quad s \in \mathbb{R}^{+} .
$$

We assume that the strain $E(\cdot)$ belongs to $C^{2}$ on $\mathbb{R}$. The stress is denoted by $T(t)$. The general form of the constitutive relation is

$$
T(t)=\widetilde{T}\left(E^{t}, E(t)\right),
$$

where it is understood that $\widetilde{T}$ is a functional of $E^{t}$ and a function of $E(t)$. The general form of a free energy functional is given by

$$
\psi(t)=\tilde{\psi}\left(E^{t}, E(t)\right) \geq 0,
$$

where, as with $\tilde{T}$, the quantity $\tilde{\psi}$ is a functional of $E^{t}$ and a function of $E(t)$. Let $E^{\dagger}$ be the static history, equal to $E(t)$ at the current and all past times. Then

$$
\tilde{\psi}\left(E^{\dagger}, E(t)\right)=\widetilde{\phi}(E(t))=\phi(t) \geq 0
$$

where $\vec{\phi}(E(t))$ is the equilibrium free energy. This is a definition of $\widetilde{\phi}$, which must have the same form for any choice of free energy. The abbreviation to $\phi(t)$ will be used frequently.

$$
\text { "161_2016_512_Article" — 2016/6/15 - 9:11 — page 2 - \#2 }
$$




\subsection{Required properties of a free energy}

We denote a particular free energy at time $t$ by $\psi(t)=\tilde{\psi}\left(E^{t}, E(t)\right)$. Certain properties of free energies, derived in [5], have been used to characterize such quantities in $[8,11,14]$ and elsewhere. These are now listed.

P1: We have

$$
\frac{\partial \tilde{\psi}\left(E^{t}, E(t)\right)}{\partial E(t)}=\frac{\partial \psi(t)}{\partial E(t)}=T(t)=\widetilde{T}\left(E^{t}, E(t)\right) .
$$

Thus, any choice of free energy must yield the same $\widetilde{T}$, according to this prescription.

P2: For any history and current value $\left(E^{t}, E(t)\right)$,

$$
\bar{\psi}\left(E^{t}, E(t)\right) \geq \bar{\phi}(E(t)),
$$

where equality is achieved for the static history $E^{\dagger}$

P3: We have

$$
\dot{\psi}(t)+D(t)=T(t) \dot{E}(t), \quad D(t) \geq 0,
$$

where $D(t)$ is the rate of energy dissipation associated with $\psi(t)$. The first relation is a statement of the first law, while the non-negativity of $D(t)$ is in effect the second law.

These are a version of the Graffi conditions for a free energy [23]. Note that properties P1 and P2 follow from the second law, which is included in $\mathrm{P} 3[5,19]$

Let us assume that $\lim _{t \rightarrow-\infty} \psi(t)=0$. Then, integrating (2.8) over all past history, we obtain

$$
\psi(t)+\mathcal{D}(t)=W(t),
$$

where $\mathcal{D}(t)$ is the total dissipation in the material up to time $t$, defined by

$$
\mathcal{D}(t)=\widetilde{\mathcal{D}}\left(E^{t}, E(t)\right)=\int_{-\infty}^{t} D(u) \mathrm{d} u \geq 0,
$$

while $W(t)$ is the work function, given by

$$
W(t)=\int_{-\infty}^{t} T(u) \dot{E}(u) \mathrm{d} u=\widetilde{W}\left(E^{t}, E(t)\right),
$$

if the integrals in (2.10) and (2.11) exist. Note that

$$
\mathcal{D}(t)=D(t), \quad \dot{W}(t)=T(t) \dot{E}(t) .
$$

The quantity $W(t)$ behaves similarly to a free energy functional with zero dissipation associated with it (for example $[1,19]$ ), in the traditional sense that it obeys P1-P3. From $(2.9)$, we have in general the property that

$$
\psi(t) \leq W(t) .
$$

\section{Constitutive equations and periodicity}

Let $T(t)$ be the stress at time $t$. Then the constitutive relations with linear memory terms have the equivalent forms

$$
\begin{aligned}
T(t) & =T_{\mathrm{e}}(t)+\int_{0}^{\infty} G^{\prime}(u) E_{r}^{t}(u) \mathrm{d} u \\
& =T_{0}(t)+\int_{0}^{\infty} G^{\prime}(u) E^{t}(u) \mathrm{d} u \\
& =T_{\mathrm{e}}(t)+\int_{0}^{\infty} \tilde{G}(u) \dot{E}^{t}(u) \mathrm{d} u, \\
T_{0}(t) & =T_{\mathrm{e}}(t)+\left(G_{0}-G_{\infty}\right) E(t), \\
G^{\prime}(u) & =\frac{\mathrm{d}}{\mathrm{d} u} G(u), \quad \widetilde{G}(u)=G(u)-G_{\infty}, \\
\dot{E}^{t}(u) & =\frac{\partial}{\partial t} E^{t}(u)=-\frac{\partial}{\partial u} E^{t}(u)=-\frac{\partial}{\partial u} E_{r}^{t}(u),
\end{aligned}
$$


where $T_{\mathrm{e}}(t)$ is the stress function for the equilibrium limit $\left(E_{r}^{t}(u)=0, \quad u \in \mathbb{R}^{+}\right.$) and the quantity $G(\cdot)$ $\mathbb{R}^{+} \mapsto \mathbb{R}^{+}$is the relaxation function of the material. We define

$$
G_{\infty}=G(\infty), \quad G_{0}=G(0), \quad \widetilde{G}(0)=G_{0}-G_{\infty}=\widetilde{G}_{0} .
$$

The assumption is made that

$$
\widetilde{G}, G^{\prime} \in L^{1}\left(\mathbb{R}^{+}\right) \cap L^{2}\left(\mathbb{R}^{+}\right),
$$

which will be relevant in the context of taking the Fourier transform of these quantities. The quantity $T_{\mathrm{e}}(t)$ in (3.1) is given by

$$
T_{\mathrm{e}}(t)=\frac{\partial \phi(t)}{\partial E(t)},
$$

which is the static limit of (2.6). For a completely linear material,

$$
\tilde{\phi}(E(t))=\frac{1}{2} G_{\infty} E^{2}(t) .
$$

and $T_{\mathrm{e}}(t), T_{0}(t)$ are given by the forms

$$
T_{\mathrm{e}}(t)=G_{\infty} E(t), \quad T_{0}(t)=G_{0} E(t) .
$$

However, more general choices of $\phi(t)$ are also possible, leading to non-linear forms of $T_{\mathrm{e}}(t)$. It will be assumed in the present work that (3.6) holds

Relation (3.1) 3 can be expressed in the following alternative form, by a change of integration variable:

$$
T(t)=T_{\mathrm{e}}(t)+\int_{-\infty}^{t} \tilde{G}(t-s) \dot{E}(s) \mathrm{d} s .
$$

A similar remark applies to $(3,1)_{1,2}$. The form (3.7) is convenient for considering the case of histories which are zero for negative times, i.e. $E(s)=0, s<0$. Such histories are of interest below. Under this assumption, (3.7) becomes

$$
T(t)= \begin{cases}T_{\mathrm{e}}(t)+\int_{0}^{t} \widetilde{G}(t-s) \dot{E}(s) \mathrm{d} s, & t \geq 0, \\ 0, & t<0 .\end{cases}
$$

\subsection{Periodic histories for general materials}

Some of the arguments in this subsection were first given in [1], page 116.

Consider periodic histones over $\mathbb{R}$, giving

$$
E\left(t+t_{\mathrm{p}}\right)=E(t), \quad t \in \mathbb{R},
$$

where $t_{\mathrm{p}}$ is the period. Differentiating with respect to $t$, we obtain

$$
\dot{E}\left(t+t_{\mathrm{p}}\right)=\dot{E}(t) .
$$

It is assumed that $\psi(t)$, given by (2.4), is finite. It is probably a general consequence of this that $\psi(t)$ is fully periodic, but in any case, this is shown to be true in Sects. 9.1 and 11.2 for quadratic free energy functionals. We therefore assert that

$$
\psi\left(t+t_{\mathrm{p}}\right)=\psi(t), \quad \dot{\psi}\left(t+t_{\mathrm{p}}\right)=\dot{\psi}(t), \quad t \in \mathbb{R} .
$$

By virtue of $\mathrm{P} 1$ or (2.6),

$$
T\left(t+t_{\mathrm{p}}\right)=\frac{\partial \psi\left(t+t_{\mathrm{p}}\right)}{\partial E\left(t+t_{\mathrm{p}}\right)}=\frac{\partial \psi(t)}{\partial E(t)}=T(t) .
$$

From (2.8), it follows that

$$
D\left(t+t_{\mathrm{p}}\right)=D(t) .
$$

Integrating (2.8) over $\left[t, t+t_{p}\right]$, we obtain

$$
\int_{t}^{t+t_{\mathrm{p}}} D(u) \mathrm{d} u=\int_{t}^{t+t_{\mathrm{p}}} T(u) \dot{E}(u) \mathrm{d} u>0
$$


for materials with dissipation, or

$$
\mathcal{D}\left(t+t_{\mathrm{p}}\right)-\mathcal{D}(t)=W\left(t+t_{\mathrm{p}}\right)-W(t)>0 .
$$

Now

$$
\frac{\mathrm{d}}{\mathrm{d} t}\left[W\left(t+t_{\mathrm{p}}\right)-W(t)\right]=T\left(t+t_{\mathrm{p}}\right) \dot{E}\left(t+t_{\mathrm{p}}\right)-T(t) \dot{E}(t)=0,
$$

so that both sides of (3.14) and (3.15) are independent of $t$. The infinite integral defining $\mathcal{D}(t)$ in $(2.10)$ and $W(t)$ in (2.11) therefore must diverge for periodic histories since they consist of an infinite sum of integrals over a period, as in (3.14), each being non-zero and equal to all the others.

The divergences in $\mathcal{D}(t)$ and $W(t)$ must cancel if $\psi(t)$ is to be finite.

Remark 3.I The relation (3.11) $)_{1}$ will be regarded as a basic property of any free energy functional, sufficiently important to label it as P4, following P3, defined by (2.8).

Therefore, we have

P4: For a long established petiodic history, where transient effects have died away, a free energy functional must obey the condition

$$
\psi\left(t+t_{\mathrm{p}}\right)=\psi(t), \quad t \in \mathbb{R} .
$$

where $t_{\mathrm{p}}$ is the core period. From (3.15) it follows that (3.17) does not hold for $W(t)$ if the material is dissipative.

The special property of $\psi(t)$, not shared by $\mathcal{D}(t)$ and $W(t)$, that it is finite for infinite periodic histories is a reflection of the fact that it is related to energy stored in the body, which must be finite. In particular, the minimum free energy, for a given state, is equal to the maximum recoverable work from that state.

\subsection{Convexity property of free energies}

This fundamental property gives that if $\psi_{1}(t)$ and $\psi_{2}(t)$ are free energies, then

$$
\psi(t)=a \psi_{1}(t)+b \psi_{2}(t), \quad a, b \in \mathbb{R}^{+}, \quad a+b=1,
$$

is also a free energy. It is straightforward to check that if $\psi_{1}(t)$ and $\psi_{2}(t)$ obey properties P1-P4, then $\psi(t)$ also has these properties.

\section{The general form of a free energy associated with linear memory}

For a scalar theory with a linear memory constitutive relation, the most general form of a free energy is

$$
\begin{aligned}
\psi(t) & =\phi(t)+\frac{1}{2} \int_{0}^{\infty} \int_{0}^{\infty} E_{r}^{t}(s) \mathcal{G}(s, u) E_{r}^{t}(u) \mathrm{d} s \mathrm{~d} u \\
& =S(t)+\frac{1}{2} \int_{0}^{\infty} \int_{0}^{\infty} E^{t}(s) \mathcal{G}(s, u) E^{t}(u) \mathrm{d} s \mathrm{~d} u \\
& =\phi(t)+\frac{1}{2} \int_{0}^{\infty} \int_{0}^{\infty} \dot{E}^{t}(s) \widetilde{G}(s, u) \dot{E}^{t}(u) \mathrm{d} s \mathrm{~d} u, \\
\mathcal{G}(s, u) & =\frac{\partial^{2}}{\partial s \partial u} G(s, u)=G_{12}(s, u), \\
\vec{G}(s, u) & =G(s, u)-G_{\infty}, \quad s, u \in R^{+},
\end{aligned}
$$

where $S(t)$, for completely linear materials ([1], page 134), is given by

$$
S(t)=T(t) E(t)-\frac{1}{2} G_{0} E^{2}(t) .
$$


There is no loss of generality in taking

$$
\widetilde{G}(s, u)=\widetilde{G}(u, s), \quad \mathcal{G}(s, u)=\mathcal{G}(u, s) .
$$

The following properties of $G(\cdot, \cdot)$ hold ([1], chapter 6 and earlier references therein):

$$
\begin{gathered}
G(\infty, u)=G(s, \infty)=G_{\infty}, \\
G_{I}(s, \infty)=G_{2}(\infty, u)=0, \quad G_{1}(\infty, s)=G_{2}(u, \infty)=0 \forall s, u \in \mathbb{R}^{+} .
\end{gathered}
$$

The relaxation function $G(u)$ is given by

$$
G(u)=G(0, u)=G(u, 0) \quad \forall u \in \mathbb{R}^{+}
$$

yielding

$$
G^{\prime}(u)=G_{2}(0, u)=G_{1}(u, 0) .
$$

The Graffi condition $\mathrm{P} 2$, given by $(2.7)$, requires that the kernels $\mathcal{G}(\cdot, \cdot)$ and $\widetilde{G}(\cdot, \cdot)$ must be such that the integral terms in $(4.1)_{1,3}$ are non-negative. It follows that the integral term in $(4.1)_{2}$ is also non-negative.

Remark 4.I Note that the quantity $G(u)$ is the same for all choices of free energy, i.e. for all choices of $G(s, u)$, since it generates the defining constitutive equation for the material.

The rate of dissipation and total dissipation can be deduced from (2.8) and (2.9). These are very important in the context of discussing free energies; indeed, they are fundamental [20]. However, in the present work, we will focus on the free energy functionals, omitting details relating to $D(t)$ and $D(t)$

There are two equivalent notations, the first being to use $\widetilde{G}(s, u), \dot{E}^{t}(s)$ and the second to use $\mathcal{g}(s, u)$, $E_{r}^{t}(s)$. Both will occur in what follows, though the first option is favoured.

By a double change of variables, we can express $(4.1)_{3}$ (and analogously $\left.(4.1)_{1,2}\right)$ in the form (cf. (3.7))

$$
\psi(t)=\phi(t)+\frac{1}{2} \int_{-\infty}^{t} \int_{-\infty}^{t} \dot{E}(s) \widetilde{G}(t-s, t-u) \dot{E}(u) \mathrm{d} s \mathrm{~d} u
$$

so that in relation to histories which vanish for negative times, we have (cf. (3.8))

$$
\psi(t)= \begin{cases}\phi(t)+\frac{1}{2} \int_{0}^{t} \int_{0}^{t} \dot{E}(s) \widetilde{G}(t-s, t-u) \dot{E}(u) \mathrm{d} s \mathrm{~d} u, & t \geq 0, \\ 0, & t<0 .\end{cases}
$$

Remark 4.2 We will require a measure of distance between free energies, denoted in general by $\left\|\psi_{1}-\psi_{2}\right\|$, where $\|\cdot\|$ indicates some standard functional norm. It will be assumed that the histories of strain are non-zero only on $\mathbb{R}^{+}$, yielding functionals with the same property, as in (4.8). A simple choice is

$$
\left\|\psi_{1}-\psi_{2}\right\|=\max _{u \in \mathbb{R}^{+}}\left|\psi_{1}(u)-\psi_{2}(u)\right| .
$$

This is satisfactory for histories that converge to zero or a constant at large times. However, we will be considering exponentially growing histories, also. In this case, the time value at which the maximum occurs will tend to diverge, so that it was decided to choose a variation on (4.9) of the form

$$
\left\|\psi_{1}-\psi_{2}\right\|=\max _{u \in \mathbb{R}^{+}}\left|\frac{\psi_{1}(u)-\psi_{2}(u)}{\psi_{M}(u)}\right| .
$$

where $\psi_{M}(u)$ is the maximum free energy.

These measures of distance will be referred to below as differences or separations.

The work function $W(t)$ can be expressed in the form (4.1) by choosing ([1], page 153 and many earlier references)

$$
\widetilde{G}(s, u)=\widetilde{G}(|s-u|) .
$$




\section{Minimal states}

As noted earlier, a viscoelastic state is defined in general by the history and current value of strain $\left(E^{t}, E(t)\right)$. The term minimal state was introduced in [12], but the concept is older, being based on the work of Noll [24] and developed in for example [6-8,22]. Further references are given in [1]. Minimal states for materials with linear constitutive relations can be described as follows [6,7]: two viscoelastic states $\left(E_{1}^{t}, E_{1}(t)\right),\left(E_{2}^{t}, E_{2}(t)\right)$ are equivalent or in the same equivalence class or minimal state if

$$
\begin{aligned}
E_{1}(t) & =E_{2}(t), \\
\int_{0}^{\infty} G^{\prime}(s+\tau)\left[E_{1 r}^{t}(s)-E_{2 r}^{t}(s)\right] \mathrm{d} s & =I_{1}^{t}(\tau)-I_{2}^{t}(\tau)=0 \forall \tau \geq 0, \\
I^{t}(\tau)=\int_{0}^{\infty} G^{\prime}(s+\tau) E_{r}^{t}(s) \mathrm{d} s & =\int_{0}^{\infty} \tilde{G}(s+\tau) \dot{E}^{t}(s) \mathrm{d} s, \\
I_{i}^{t}(\tau)=\int_{0}^{\infty} G^{\prime}(s+\tau) E_{r i}^{t}(s) \mathrm{d} s & =\int_{0}^{\infty} \widetilde{G}(s+\tau) \dot{E}_{i}^{t}(s) \mathrm{d} s, \quad i=1,2 .
\end{aligned}
$$

A functional of $\left(E^{t}, E(t)\right)$ which yields the same value for all members of the same minimal state is referred to as a functional of the minimal state or a minimal state variable. The abbreviation FMS was introduced earlier

Remark 5.1 A fundamental distinction between materials ([1,9], page 342) is that tor certau. .

tions, namely those with only isolated singularities (in the frequency domain), the set of minimal slaues is non-singleton, while if some branch cuts are present in the relaxation function, the material has only singleton minimal states. For relaxation functions with only isolated singularities, there is a maximum free energy $\psi_{M}(t)$ that is less than the work function $W(t)$ and also a set of related intermediate free energies. On the other hand, if branch cuts are present, the maximum free energy is $W(t)$ [if we choose to ignore condition (3.17)], and there are no intermediate free energies other than those we shall construct in Remark 5.2 below.

The simplest case of isolated singularities occur in discrete spectrum materials (see Sect. 7.1). These are characterized by relaxation functions which, in the frequency domain, consist of a series of isolated simple poles on the positive imaginary axis. In the time domain, such relaxation functions are given by sums of strictly decaying exponentials. They are used in the present work as realistic examples of materials with isolated singularities, which avoid the complexity of the general case ([1], chapters 15 and 16).

The simplest case of non-isolated singularities is a continuous spectrum material $[1,10]$ for which the relaxation function is given by integrals of density functions multiplying strictly decaying exponentials (see Sect. 7.2). The form of $\psi_{m}(t)$ for such materials is explicitly derived in [10].

Relaxation functions for both of these materials are strictly monotonic. This need not be so for more general materials.

It can be shown that if the material has minimal states that are non-singleton then the free energy functional is positive semi-definite ([1], page 152).

A free energy $\psi(t)=\tilde{\psi}\left(E^{t}, E(t)\right)$, is a FMS if it has the property that any two members $\left(E_{1}^{t}, E(t)\right)$, $\left(E_{2}^{t}, E(t)\right)$ of the same minimal state yield equal values of $\tilde{\psi}$, or

$$
\tilde{\psi}\left(E_{1}^{t}, E(t)\right)=\tilde{\psi}\left(E_{2}^{t}, E(t)\right) \text {. }
$$

A free enetgy need not have this property, though most of those which are of interest are in fact FMSs [4]. We shall seek to impose this constraint in the present context.

The Graffi-Volterra free energy is not a FMS $[1,7]$ and therefore will not be included in our discussion. However, for the present, the functional $W(t)$, which is also not a FMS $[1,7]$, will be included, but finally dropped because, as pointed out in Remark 3.1, it does not obey (3.17).

Remark 5.2 For materials in which minimal states are singletons, we identify the maximum free energy with the work function, and so, using (3.18), give a general form of the free energy as

$$
\psi(t)=c_{1} \psi_{m}(t)+c_{2} W(t), \quad c_{1}, c_{2} \in \mathbb{R}^{+}, \quad c_{1}+c_{2}=1,
$$

where $\psi_{m}(t)$ is the minimum free energy for such materials.

For materials with strictly decaying relaxation functions (for example [1], chapter 9), the Dill and Fabrizio functionals are free energies, denoted by $\psi_{\text {Dill }}$ and $\psi_{F}$ respectively, and could be included on the right-hand

$$
\text { “161_2016_512_Article” - 2016/6/15 — 9:11 — page } 7 \text { — \#7 }
$$


side of (5.3) 1 . Also, another category of recently derived free energy functionals [18] may be included. The term proportional to $W(t)$ ensures that free energies larger than $\psi_{m}, \psi_{\text {Dill }}, \psi_{F}$ etc. are encompassed by the formula.

Leaving aside the new category discussed in [18], then, for such materials, we could generalize $(5.3)$ to

$$
\begin{aligned}
& \psi(t)=c_{1} \psi_{m}(t)+c_{2} \psi_{\text {Dill }}(t)+c_{3} \psi_{F}(t)+c_{4} W(t), \\
& c_{i} \in \mathbb{R}^{+}, i=1, \ldots, 4, \quad \sum_{j=1}^{4} c_{j}=1 .
\end{aligned}
$$

The problem with (5.3) and (5.4) is that if the coefficient of $W(t)$ is greater than zero, then the free energy $\psi(t)$ does not obey $\mathrm{P} 4$, given by (3.17).

Remark 5.3 The objective of the present work is to propose an alternative to (5.3) or (5.4)

\section{Frequency domain quantities}

Let $\Omega$ be the complex $\omega$ plane and

$$
\begin{aligned}
\Omega^{+} & =\left\{\omega \in \Omega \mid \operatorname{Im}(\omega) \in \mathbb{R}^{+}\right\}, \\
\Omega^{(+)} & =\left\{\omega \in \Omega \mid \operatorname{Im}(\omega) \in \mathbb{R}^{++}\right\} .
\end{aligned}
$$

These define the upper half-plane including and excluding the real axis, respectively. Similarly, $\Omega^{-}, \Omega^{(-)}$are the lower half-planes including and excluding the real axis, respectively.

Remark 6.1 Throughout this work, a subscript "+" attached to any quantity defined on $\Omega$ will imply that it is analytic on an open set including $\Omega^{-}$, with all its singularities in $\Omega^{(+)}$. Similarly, a subscript " - " will indicate that it is analytic on an open set including $\Omega^{+}$, with all its singularities in $\Omega^{(-)}$.

We shall abbreviate these descriptions by omitting reference to open sets, replacing them by "analytic in $\Omega^{(-) "}$ and "analytic in $\Omega^{(+)}$, , respectively. These phrases are understood to include the reference to open sets.

\subsection{Relaxation functions}

Let us define the Fourier transforms

$$
\begin{aligned}
& \widetilde{G}_{+}(\omega)=\int_{0}^{\infty} \widetilde{G}(s) \mathrm{e}^{-i \omega s} \mathrm{~d} s=\widetilde{G}_{c}(\omega)-i \widetilde{G}_{s}(\omega), \\
& G_{+}^{\prime}(\omega)=\int_{0}^{\infty} G^{\prime}(s) \mathrm{e}^{-i \omega s} \mathrm{~d} s=G_{c}^{\prime}(\omega)-i G_{s}^{\prime}(\omega) .
\end{aligned}
$$

It follows from (3.3) that $G_{+}^{\prime}$ and $\widetilde{G}_{+} \in L^{2}(\mathbb{R})$. Applying a partial integration in (6.2) 3 yields that

$$
G_{c}^{\prime}(\omega)=-\widetilde{G}_{0}+\omega \widetilde{G}_{s}(\omega), \quad G_{s}^{\prime}(\omega)=-\omega \widetilde{G}_{c}(\omega) .
$$

The equivalent inequalities $[1,11]$

$$
\widetilde{G}_{c}(\omega) \geq 0, \quad G_{s}^{\prime}(\omega) \leq 0 \quad \forall \omega \in \mathbb{R}^{++},
$$

are consequences of the second law. We have

$$
\widetilde{G}_{0}>0, \quad G_{\infty}>0,
$$

the latter relation being true for a viscoelastic solid. The functions $\widetilde{G}_{+}(\omega)$ and $G_{+}^{\prime}(\omega)$ are analytic on $\Omega^{-}([1]$, pages 547,548 ).

A quantity which will be of significant interest, particularly in the context of the minimum and related free energies, is

$$
H(\omega)=-\omega G_{s}^{\prime}(\omega)=\omega^{2} \widetilde{G}_{c}(\omega) \geq 0, \omega \in \mathbb{R},
$$

$$
\text { "161_2016_512_Article" — 2016/6/15 - 9:11 - page } 8 \text { — \#8 }
$$


where the inequality is a consequence of (6.4), and (6.3) 2 has been invoked. One can show that

$$
H_{\infty}=\lim _{\omega \rightarrow \infty} H(\omega)=-G^{\prime}(0) \geq 0
$$

We assume for present purposes that $G^{\prime}(0)$ is non-zero so that $H_{\infty}$ is a finite, positive number. Then $H(\omega) \in$ $\mathbb{R}^{++} \forall \omega \in \mathbb{R}, \omega \neq 0$.

The non-negative quantity $H(\omega)$ can always be expressed as the product of two factors

$$
H(\omega)=H_{+}(\omega) H_{-}(\omega)
$$

where $H_{+}(\omega)$ has no zeros in $\Omega^{(-)}$and is analytic in $\Omega^{-}$. Similarly, $H_{-}(\omega)$ is analytic in $\Omega^{+}$with no zeros in $\Omega^{(+)}$. We put $[1,14]$

$$
\begin{aligned}
H_{ \pm}(\omega) & =H_{\mp}(-\omega)=\overline{H_{\mp}}(\omega), \\
H(\omega) & =\left|H_{ \pm}(\omega)\right|^{2}, \quad \omega \in \mathbb{R} .
\end{aligned}
$$

A general method is outlined in $[1,14]$ for determining the factors of $H$, though for the case of discrete spectrum materials, discussed in Sect. 9.1, one can deduce $H_{ \pm}$by inspection.

The factorization (6.8) is the one relevant to the minimum free energy. For materials with only isolated singularities, there is a much broader class of factorizations, where the property that the zeros of $H_{ \pm}(\omega)$ are in $\Omega^{ \pm}$, respectively, need not be true. These generate a range of free energies related to the minimum free energy, as discussed briefly in Sect. 9.1.

Referring to (6.6), we note that $H_{ \pm}(\omega)$ go to zero linearly at the origin.

\subsection{Strain history}

The Fourier transform of $E^{t}(s)$, which is zero for $s \in \mathbb{R}^{--}$is denoted by $E_{+}^{i}(\omega)$ and is given by

$$
E_{+}^{t}(\omega)=\int_{0}^{\infty} E^{t}(s) \mathrm{e}^{-i \omega s} \mathrm{~d} s
$$

This quantity is analytic on $\Omega^{-}$. The Fourier transform of $E_{r}^{t}$, defined by (2.2) and zero for $s \in \mathbb{R}^{--}$, has the form ([1], page 548)

$$
E_{r+}^{t}(\omega)=E_{+}^{t}(\omega)-\frac{E(t)}{i \omega^{-}}
$$

The notation $\omega^{-}$along with $\omega^{+}$, which will be required below, was introduced in [14] and used in subsequent work. The notation $\omega^{ \pm}$implies that $\omega$ is slightly off the real axis at $\omega_{r} \pm i \epsilon$, respectively, where $\omega_{r}$ is on the real axis and $\epsilon>0$. The limit $\omega \rightarrow \omega_{r}$ can be taken after integrations have been carried out. Note that ([1], page 145)

$$
\frac{\mathrm{d}}{\mathrm{d} t} E_{+}^{t}(\omega)=\dot{E}_{+}^{t}(\omega)=-i \omega E_{+}^{t}(\omega)+E(t)=-i \omega E_{r+}^{t}(\omega)
$$

\section{Discrete and continuous spectrum materials}

We now list some basic formulae relating to discrete and continuous spectrum materials. These will be used in the present work as conveniently simple but realistic examples of materials with only isolated singularities and with branch cuts respectively.

$$
\text { “161_2016_512_Article” — 2016/6/15 — 9:11 — page } 9 \text { — \#9 }
$$




\subsection{Discrete spectrum formulae}

The form of the relaxation function for discrete spectrum materials is given by

$$
\widetilde{G}(s)=\sum_{i=1}^{n} G_{i} \mathrm{e}^{-\alpha_{i} s},
$$

where $n$ is a positive integer. The quantities $\alpha_{i} \in \mathbb{R}^{+}, i=1,2, \ldots, n$ are the inverse decay times, while the coefficients $G_{i}$ are also generally assumed to be positive, this being the simplest way to ensure the condition (6.4), which is clear from $(7.3)_{2}$ below. Note that

$$
G_{0}-G_{\infty}=\widetilde{G}_{0}=\sum_{i=1}^{n} G_{i}
$$

Let us arrange that $\alpha_{1}<\alpha_{2}<\alpha_{3} \ldots$ From (6.2)1, we have

$$
\widetilde{G}_{+}(\omega)=\sum_{i=1}^{n} \frac{G_{i}}{\alpha_{i}+i \omega}, \quad \widetilde{G}_{c}(\omega)=\sum_{i=1}^{n} \frac{\alpha_{i} G_{i}}{\alpha_{i}^{2}+\omega^{2}}, \quad \widetilde{G}_{s}(\omega)=\omega \sum_{i=1}^{n} \frac{G_{i}}{\alpha_{i}^{2}+\omega^{2}} .
$$

Observe that the singularities of $\widetilde{G}_{+}(\omega)$ consist of simple poles at $\omega=i \alpha_{i}, i=1,2, \ldots, n$. Relation (6.6) gives

$$
H(\omega)=\omega^{2} \sum_{i=1}^{n} \frac{\alpha_{i} G_{i}}{\alpha_{i}^{2}+\omega^{2}} \geq 0 .
$$

The limit of $H(\omega)$ for large $\omega$, introduced in (6.7), becomes

$$
H_{\infty}=\sum_{i=1}^{n} \alpha_{i} G_{i}
$$

We have [14]

$$
H(\omega)=H_{\infty} \prod_{i=1}^{n}\left\{\frac{\gamma_{i}^{2}+\omega^{2}}{\alpha_{i}^{2}+\omega^{2}}\right\},
$$

where the $\gamma_{i}^{2}$ are the zeros of $f(z)=H(\omega), z=-\omega^{2}$ and obey the relations

$$
\gamma_{1}=0, \quad \alpha_{1}^{2}<\gamma_{2}^{2}<\alpha_{2}^{2}<\gamma_{3}^{2} \ldots
$$

Let us now characterize minimal states in the context of discrete spectrum materials. Consider two states $\left(E_{1}^{t}, E_{1}(t)\right)$ and $\left(E_{2}^{t}, E_{2}(t)\right)$ obeying conditions (5.1), so that they are equivalent. We define the difference between these states as $\left(E_{d}^{t}, E_{d}(t)\right)$ where

$$
E_{d}^{t}(s)=E_{1}^{t}(s)-E_{2}^{t}(s) \quad \forall s \in R^{\dagger}, \quad E_{d}(t)=E_{1}(t)-E_{2}(t) .
$$

The conditions (5.1) holds for all $\tau \geq 0$ if and only if

$$
\begin{gathered}
E_{d}(t)=0, \quad \int_{0}^{\infty} \mathrm{e}^{-\alpha_{i} s} E_{d}^{t}(s) \mathrm{d} s=E_{d+}^{t}\left(-i \alpha_{i}\right)=0, \\
i=1,2, \ldots, n,
\end{gathered}
$$

Remark 7.1 Thus, for a given discrete spectrum material, the property that two given histories are equivalent, or in the same minimal state, is determined solely by the values of those histories in the frequency domain at $\omega=-i \alpha_{i}, i=1,2, \ldots, n$.

For discrete spectrum materials, the kernel $\widetilde{G}(\cdot \cdot)$, introduced in (4.1), has the form [17]

$$
\widetilde{G}(s, u)=\sum_{i, j=1}^{n} C_{i j} \mathrm{e}^{-\alpha_{j} s-\alpha_{j} u}
$$

where $C_{i j}$ are the components of a positive definite matrix $\mathbf{C}$.

$$
\text { “161_2016_512_Arlicle" - 2016/6/15 - 9:11 - page 10 - \#10 }
$$


7.2 Continuous spectrum formulae

The form of the relaxation function for a continuous spectrum material is given by

$$
\widetilde{G}(s)=\int_{a}^{b} g(\alpha) \mathrm{e}^{-\alpha s} \mathrm{~d} \alpha, \quad g(\alpha) \geq 0 \forall \alpha \in[a, b], \quad b>a,
$$

where $a$ and $b$ are two real, positive numbers, so that

$$
\begin{aligned}
& \widetilde{G}_{+}(\omega)=\int_{a}^{b} \frac{g(\alpha)}{\alpha+i \omega} \mathrm{d} \alpha, \\
& \widetilde{G}_{c}(\omega)=\int_{a}^{b} \frac{\alpha g(\alpha)}{\alpha^{2}+\omega^{2}} \mathrm{~d} \alpha, \quad \widetilde{G}_{s}(\omega)=\omega \int_{a}^{b} \frac{g(\alpha)}{\alpha^{2}+\omega^{2}} \mathrm{~d} \alpha .
\end{aligned}
$$

The singularity structure of $\widetilde{G}_{+}(\omega)$ consists of a single branch cut on $[a, b]$. Relation (6.6) gives that

$$
H(\omega)=\omega^{2} \int_{a}^{b} \frac{\alpha g(\alpha)}{\alpha^{2}+\omega^{2}} \mathrm{~d} \alpha \geq 0
$$

Minimal states in the context of continuous spectrum materials are singletons [10].

The theory developed in [1], chapter 16 generalizes (7.3) to materials with isolated singularities of different types. Relation $(7.12)_{1}$ can be generalized also to describe materials with other types of branch cuts.

\section{Approximating continuous spectrum behavior by discrete spectrum formulae}

The form of the minimum free energy for continuous spectrum materials was discussed in detail in [10], with explicit formulae given for all quantities of interest. We wish to explore another approach here, which is to approximate the continuous spectrum case by a discrete spectrum material.

The quantity $H(\omega)$, given by (7.13), can be approximated by a quadrature formula

$$
H(\omega)=\omega^{2} \sum_{i=1}^{n} \frac{\alpha_{i} \kappa_{i} g\left(\alpha_{i}\right)}{\alpha_{i}^{2}+\omega^{2}}
$$

where the constants $\alpha_{i}, i=1,2, \ldots, n$ are values of $\alpha \in[a, b]$ and $\kappa_{i}$ are the quadrature coefficients associated with the particular method chosen (trapezoidal, Simpson, Gauss etc.). Comparing with (7.4), we put

$$
G_{i}=\kappa_{i} g\left(\alpha_{i}\right), \quad i=1,2, \ldots, n
$$

to obtain a discrete spectrum material approximating the continuous spectrum material under discussion.

In the numerical work described in Sect. 13, we use the simplest option, namely the trapezoidal rule, so that

$$
\begin{aligned}
& \alpha_{i}=a+\frac{(i-1)(b-a)}{n-1}, \quad i=1,2, \ldots, n, \\
& \kappa_{i}= \begin{cases}\frac{b-a}{n-1}, & i=2,3, \ldots, n-1, \\
\frac{b-a}{2(n-1)}, & i=1, n .\end{cases}
\end{aligned}
$$




\section{The minimum and related free energies}

It is shown in $[1,12,15]$ that, for materials with only isolated singularities, the quantity $H(\omega)$ is a rational function (see, for example, (7.6)) and has many factorizations other than (6.8), denoted by

$$
H(\omega)=H_{+}^{f}(\omega) H_{-}^{f}(\omega), \quad H_{ \pm}^{f}(\omega)=H_{\mp}^{f}(-\omega)=\overline{H_{\mp}^{f}}(\omega),
$$

where $f$ is an identification label distinguishing a particular factorization. These are obtained by exchanging the zeros of $H_{+}(\omega)$ and $H_{-}(\omega)$, leaving the singularities unchanged. For any $f$, the work function can be expressed as ([1], page 353)

$$
\begin{aligned}
W(t) & =\phi(t)+\frac{1}{2 \pi} \int_{-\infty}^{\infty}\left[\left|p_{-}^{f t}(\omega)\right|^{2}+\left|p_{+}^{f t}(\omega)\right|^{2}\right] \mathrm{d} \omega, \\
p_{ \pm}^{f t}(\omega) & =\frac{1}{2 \pi i} \int_{-\infty}^{\infty} \frac{H_{-}^{f}\left(\omega^{\prime}\right) E_{r+}^{f}\left(\omega^{\prime}\right)}{\omega^{\prime}-\omega^{\mp}} \mathrm{d} \omega^{\prime}=\frac{1}{2 \pi} \int_{-\infty}^{\infty} \frac{H_{-}^{f}\left(\omega^{\prime}\right) \dot{E}_{+}^{t}\left(\omega^{\prime}\right)}{\omega^{\prime}\left(\omega^{\prime}-\omega^{\mp}\right)} \mathrm{d} \omega^{\prime},
\end{aligned}
$$

where (6.12) has been invoked. Also, each factorization yields a different free energy of the form

$$
\psi_{f}(t)=\phi(t)+\frac{1}{2 \pi} \int_{-\infty}^{\infty}\left|p_{-}^{f t}(\omega)\right|^{2} \mathrm{~d} \omega .
$$

These various formulae apply in particular to the case where no exchange of zeros takes place, which is denoted by $f=1$. In this case, the formulae in fact apply to all materials, not just those with isolated singularities.

We can also write $\psi_{f}(t)$ as quadratic functionals in the frequency domain $[1,15]$, which are special cases of general formulae given in [17]. These latter quantities are the frequency versions of (4.1)1,3.

All these free energies are FMSs ([1], page 352). The factorization (6.8) yields the minimum free energy $\psi_{m}(t)$. Each exchange of zeros, starting from these factors, can be shown to yield a free energy which is greater than or equal to the previous quantity ([1], page 363).

Remark 9.1 A particularly interesting one is obtained by interchanging all the zeros. This can be identified as the maximum free energy among all those that are FMSs. It is less than the work function, which, as noted earlier, is not a FMS for materials with only isolated singularities.

If there are $M$ different factorizations of $H(\omega)$, then $f=M$ will be chosen for the maximum free energy, which we denote by $\psi_{M}(t)$.

\subsection{Explicit forms of the minimum and related free energies for discrete spectrum materials}

To obtain the minimum free energy, one chooses the factorization of (7.6) given by

$$
\begin{aligned}
& H_{+}(\omega)=h_{\infty} \prod_{i=1}^{n}\left\{\frac{\omega-i \gamma_{i}}{\omega-i \alpha_{i}}\right\}, \\
& H_{-}(\omega)=h_{\infty} \prod_{i=1}^{n}\left\{\frac{\omega+i \gamma_{i}}{\omega+i \alpha_{i}}\right\}, \quad h_{\infty}=\left[H_{\infty}\right]^{1 / 2},
\end{aligned}
$$

where the $\gamma_{i}$ are all non-negative. We put $\gamma_{1}=0$, which ensures that the factors $H_{ \pm}(\omega)$ go to zero linearly at the origin, as noted at the end of Sect. 6.1. Interchanging the zeros means in effect switching a given $\gamma_{i}$ to $-\gamma_{i}, i=2,3, \ldots, n$, in both $H_{+}$and $H_{-}$. Let us introduce an $n-1$-dimensional vector $\epsilon_{i}^{f}, i=2,3, \ldots, n$ where each $\epsilon_{i}^{f}$ can take values \pm 1 . There are

possible choices of $\epsilon_{i}^{f}$.

$$
M=2^{n-1}
$$

Remark 9.2 A convenient algorithm to generate the $\epsilon_{i}^{f}, i=2,3, \ldots, n$, is as follows. For each integer $f$, express $f-1 \in[0, M-1]$ as a binary number of length $n-1$, and regard each digit of this number as a component of the matrix. Change each 0 in the matrix to -1 , which completes the construction.

This method is used to produce the numerical results of Sect. 13.

$$
\text { "161_2016_512_Article" — 2016/6/15 - 9:11 — page } 12 \text { — \#12 }
$$


The free energies generated by the procedure outlined in Remark 9.2 will not be in monotonically increasing order, In particular, the free energy generated by the first row of the matrix is the maximum free energy, while that generated by the last row is the minimum free energy $\psi_{m}(t)$, taken usually to correspond to $f=1$. For any given value of $t$, they can if necessary be sorted into increasing order. In particular, the convention that $f=1$ corresponds to the minimum free energy, and $f=M$ to the maximum free energy will be maintained in the discussion below.

We define $\rho_{i}^{f}=\epsilon_{i}^{f} \gamma_{i}$, and write

$$
\begin{aligned}
& H_{+}^{f}(\omega)=h_{\infty} \prod_{i=1}^{n}\left\{\frac{\omega-i \rho_{i}^{f}}{\omega-i \alpha_{i}}\right\}, \\
& H_{-}^{f}(\omega)=h_{\infty} \prod_{i=1}^{n}\left\{\frac{\omega+i \rho_{i}^{f}}{\omega+i \alpha_{i}}\right\}
\end{aligned}
$$

Certain results from [17] and earlier references are now recalled. Equations (9.6) can be written as

$$
\begin{aligned}
H_{-}^{f}(\omega) & =i h_{\infty} \omega \sum_{i=1}^{n} \frac{R_{i}^{f}}{\alpha_{i}\left(\alpha_{i}-i \omega\right)}, \quad H_{+}^{f}(\omega)=\bar{H}_{-}^{f}(\omega), \\
R_{i}^{f} & =\left(\rho_{i}^{f}-\alpha_{i}\right) \prod_{\substack{j=1 \\
j \neq i}}^{n}\left\{\frac{\rho_{j}^{f}-\alpha_{i}}{\alpha_{j}-\alpha_{j}}\right\} .
\end{aligned}
$$

We have the formulae

$$
G_{i}=2 H_{\infty} \sum_{j=1}^{n} \frac{R_{i}^{f} R_{j}^{f}}{\left(\alpha_{i}+\alpha_{j}\right) \alpha_{i} \alpha_{j}}, \quad i=1,2, \ldots, n
$$

In the context of the present work, the quantities $G_{j}$ are related to the density function $g(\alpha)$ by $(8.2)$. The quantity $p_{-}^{f t}(\omega)$, given by $(9.2)_{2,3}$, has the form

$$
\begin{aligned}
p_{-}^{f t}(\omega) & =-i h_{\infty} \sum_{i=1}^{n} \frac{R_{i}^{f} e_{i}(t)}{\alpha_{i}\left(\omega+i \alpha_{i}\right)}, \\
e_{i}(t) & =\int_{0}^{\infty} \mathrm{e}^{-\alpha_{i} s} \dot{E}^{t}(s) \mathrm{d} s=\frac{\mathrm{d}}{\mathrm{d} t} E_{+}^{t}\left(-i \alpha_{i}\right) \\
& =-\alpha_{i} E_{r+}^{t}\left(-i \alpha_{i}\right), \quad i=1,2 \ldots, n,
\end{aligned}
$$

where (6.12) has been used. It follows from (3.10) that, for periodic histories,

$$
e_{i}\left(t+t_{\mathrm{p}}\right)=e_{i}(t), \quad i=1,2, \ldots, n .
$$

This can be deduced from the integral definition of $e_{i}(t)$ in (9.9). It follows from (9.9) and (9.3) that

$$
\psi_{f}(t)=\phi(t)+H_{\infty} \sum_{i, j=1}^{n} \frac{R_{i}^{f} R_{j}^{f}}{\left(\alpha_{i}+\alpha_{j}\right) \alpha_{i} \alpha_{j}} e_{i}(t) e_{j}(t),
$$

where the reality of the quantities $e_{i}(t)$ has been used. The property $\mathrm{P} 4$, given by $(3.17)$, follows in this case from (9.10).

If the matrix $\mathbf{C}$ in (7.10) relating to $\psi_{f}(t)$ is denoted by $\mathbf{C}^{f}$, then we have

$$
C_{i j}^{f}=2 H_{\infty} \frac{R_{i}^{f} R_{j}^{f}}{\alpha_{i} \alpha_{j}\left(\alpha_{i}+\alpha_{j}\right)} .
$$


9.2 The minimum free energy for continuous spectrum materials

The minimum free energy and related quantities were derived in [10] (see also chapter 13 of [1]). The form $(4.1)_{1}$ was used in these references. We will use (4.1) 3 here, giving

$$
\begin{aligned}
\psi_{m}(t) & =\phi(t)+\frac{1}{2} \int_{0}^{\infty} \int_{0}^{\infty} \dot{E}^{t}(s) \tilde{G}_{m}(s, u) \dot{E}^{t}(u) \mathrm{d} s \mathrm{~d} u, \\
\widetilde{G}_{m}(s, u) & =\frac{1}{2 \pi^{2}} \int_{a}^{b} \int_{a}^{b} \frac{\Delta(\alpha) \mathrm{e}^{-\alpha s} \Delta(\beta) \mathrm{e}^{-\beta s}}{(\alpha+\beta) \alpha \beta} \mathrm{d} \alpha \mathrm{d} \beta,
\end{aligned}
$$

where

$$
\begin{aligned}
& \Delta(\alpha)=\pi h_{\infty} \frac{\alpha g(\alpha)}{K(\alpha) Q(\alpha)}, \\
& K(\alpha)=-\int_{a}^{b} \frac{\beta g(\beta)}{\beta+\alpha} \mathrm{d} \beta, \quad \alpha \notin[-b,-a], \\
& L(\beta)=-P \int_{a}^{b} \frac{\lambda g(\lambda)}{\lambda-\beta} \mathrm{d} \lambda, \quad \beta \in(a, b), \\
& Q(\alpha)=\exp \left\{-\frac{1}{\pi} \int_{a}^{b} \frac{A(\beta)}{\beta+\alpha} \mathrm{d} \beta\right\} . \\
& A(\beta)=\arg \left[1-\frac{K(\beta)}{L(\beta)+i \pi \alpha g(\beta)}\right],-\pi \leq A(\beta) \leq 0 .
\end{aligned}
$$

The symbol $P$ indicates a principal value integral. If we evaluate $(9.13)_{2}$ by quadrature, the result is

$$
\widetilde{G}_{m}(s, u)=\frac{1}{2 \pi^{2}} \sum_{i, j=1}^{n} \frac{\kappa_{i} \Delta\left(\alpha_{i}\right) \kappa_{j} \Delta\left(\alpha_{j}\right)}{\left(\alpha_{i}+\alpha_{j}\right) \alpha_{i} \alpha_{j}} \mathrm{e}^{-\alpha_{i} s-\alpha_{j} u},
$$

where the $\kappa_{i}$ were introduced in (8.1), and specified for the trapezoidal rule by (8.3). Since the range of $\alpha$ and $\beta$ in (9.13) are both $[a, b]$, which is divided into $n-1$ intervals, we have replaced $\beta_{j}$ by $\alpha_{j}$ in (9.15). Comparing (9.15) with (9.12) for $f=1$ and invoking (7.10), we see that

$$
\frac{\kappa_{i} \Delta\left(\alpha_{i}\right)}{\sqrt{2} \pi}=\sqrt{2} h_{\infty} R_{i},
$$

where the quantities $R_{i}$ are the $R_{i}^{f}$ for $f=1$. The validity of this relation will be demonstrated for particular material parameters and strain histories in Sect. 13.

\subsection{The Dill and Fabrizio free energies}

The forms of these functionals are now presented. They are not obviously connected with the minimum and related free energies, discussed above. However, it emerges that for materials and histories of interest here, they are numerically close to the minimum free energy.

The Dill free energy: Opting for the notation (4.1) 3 rather than $(4.1)_{1}$ which is used in [1], we put

$$
\psi_{\text {Dill }}(t)=\phi(t)+\frac{1}{2} \int_{0}^{\infty} \int_{0}^{\infty} \tilde{G}\left(s_{1}+s_{2}\right) \dot{E}^{t}\left(s_{2}\right) \dot{E}^{t}\left(s_{1}\right) \mathrm{d} s_{1} \mathrm{~d} s_{2}
$$

which is a free energy if and only if $G(\cdot)$ is completely monotonic, as defined in [6]. This is more restrictive than (9.21) below. If $E(u)=0$ for $u<0$, relation (4.8) gives that

$$
\begin{gathered}
\psi_{\text {Dill }}(t)= \begin{cases}\phi(t)+\frac{1}{2} \int_{0}^{t} \int_{0}^{t} \dot{E}(s) \tilde{G}(2 t-s-u) \dot{E}(u) \mathrm{d} s \mathrm{~d} u, & t \geq 0, \\
0, & t<0 .\end{cases} \\
\text { “161_2016_512_Article”-2016/6/15 - 9:11 — page } 14-\# 14
\end{gathered}
$$


Free energies for singleton minimal states

The functional $\psi_{F}$ : This quantity is given by

$$
\psi_{F}(t)=\phi(t)-\frac{1}{2} \int_{0}^{\infty} \frac{\left[\dot{I}^{\prime}(\tau)\right]^{2}}{G^{\prime}(\tau)} \mathrm{d} \tau, \quad \dot{I}^{t}(\tau)=\frac{\mathrm{d}}{\mathrm{d} \tau} I^{t}(\tau),
$$

where $I^{l}(\tau)$ is defined by $(5.1)_{6}$. If $\dot{E}(u)$ is equal to zero for $u<0$,

$$
I^{t}(\tau)= \begin{cases}\int_{0}^{t} \widetilde{G}(t-u+\tau) \dot{E}(u) \mathrm{d} u, & t \geq 0, \\ 0, & t<0 .\end{cases}
$$

The quantity $\psi_{F}(t)$ is a free energy only if the conditions

$$
G^{\prime}(s) \leq 0, \quad G^{\prime \prime}(s) \geq 0, \quad \forall s \in \mathbb{R}^{+}
$$

hold.

Relations (9.21) are clearly true for the forms of the relaxation function relating to discrete and continuous spectrum materials, as given by (7.1) and (7.11).

\section{Proposed method for approximating free energies for materials with singleton minimal states}

As noted earlier, it follows from (9.10) that all the functionals $\psi_{f}(t)$ obey (3.17). This topic is further discussed in Sect. 11.2 below. The most general free energy arising from these quantities is given by $[3,15]$

$$
\psi(t)=\sum_{f=1}^{M} \lambda_{f} \psi_{f}(t), \quad \sum_{f=1}^{M} \lambda_{f}=1, \quad \lambda_{f} \geq 0 .
$$

The central proposal of this work is that we can approximate continuous spectrum materials as discrete spectrum materials, by means of (8.1). Arbitrary free energies greater than or equal to the minimum free energy are approximated by (10.1).

We need to confirm that this approximation improves as $n$ gets larger, so that there is a convergent process for increasing $n$. The value of $n$ finally chosen to approximate the continuous spectrum material should be such that convergence is essentially complete, yielding a virtually unique choice of $\psi(t)$. Based on the numerical exploration described in Sect. 13, it will be taken to be 8. For this value of $n$, there are 126 free energies $\psi_{f}(t)$ lying between $\psi_{m}(t)$ and $\psi_{M}(t)$, and indeed those given by (10.1).

Remark 10.1 It is asserted that all these free energies can be treated as free energy functionals of the continuous spectrum material. The major difficulty with this assertion is that if some other value than $n=8$ is chosen, for example, $n=9$, the intermediate free energies $\psi_{f}(t), f=2,3, \ldots, M-1$ are given by quite different formulae. However, the minimum and maximum free energies converge to fixed functionals. Numerical confirmation that this is true to a good approximation is presented in Sect. 13.

Thus, while the quantities $\psi(t)$, given by (10.1) for $n=9$, will be based on different and more numerous choices of $\psi_{f}(t)$, they are functionals spanning the same range of values as for $n=8$, providing a denser coverage of this range.

With the aim of simplifying the model to resemble (5.3), we also explore the extent to which, for each integer $f \in[2, M-1]$,

$$
\psi_{f}(t) \longrightarrow x \psi_{m}(t)+(1-x) \psi_{M}(t), \quad x \in[0,1],
$$

for large $n$. This would imply that $(10.1)_{1}$ approaches

$$
\psi(t)=c_{1} \psi_{m}(t)+c_{2} \psi_{M}(t), \quad c_{1}+c_{2}=1 .
$$

Numerical evidence was presented in [3] that $\psi_{M}(t)$ rapidly approaches $W(t)$, as $n$ gets larger, for increasing exponential histories, a result that provides good motivation for (10.3), in that it would closely approximate (5.3). However, a more extensive comparison of $\psi_{M}(t)$ and $W(t)$ is presented in Sects. 12 and 13, confirming the earlier conclusion, but also pointing to other histories for which it is not true. Thus, the relationship between (10.3) and (5.3) is not entirely simple.

$$
\text { "161_2016_512_Article" — 2016/6/15 - 9:11 — page 15 — \#15 }
$$


A significant advantage of (10.3) over (10.1) is that it matters little what $n$ we choose for the point of convergence, as long as it is above a certain minimum, say 7. Also, the argument given in Remark 10.1 is irrelevant.

The model used for this work is that described in Sect. 8, using the explicit formulae summarized in Sect. 9.1. Certain results emerge from the numerical work in Sect. 13 which support the validity of the approach, as noted in Remark 10.1. Also, (10.2) is tested and found to be a good but not a very close approximation.

Remark 10.2 The domains of definition of the $\psi_{f}(t)$ in (10.1) are greater than that of $W(t)$ in (5.3) in the sense that for certain histories, the quantities $\psi_{f}(t)$ are finite while $W(t)$ is not. The simplest examples are periodic histories, as discussed in Sect. 3.1 and in Sect. 11 below. This has consequences for existence, uniqueness and stability proofs relating to the governing dynamical equations of the materials of interest. Such proofs are an important application for explicit free energy functionals. If these use a free energy of the form (10.1) rather than that given by (5.3), the results have wider applicability.

It must, however, be noted that the minimum free energy $\psi_{m}(t)$, and indeed $\psi_{F}(t)$, given by (9.19), have particularly large domains of definition, as observed in [1], pages 222 and 276; the latter quantity is perhaps most convenient for this application.

Remark 10.3 The functionals $\psi_{\text {Dill }}(t)$ and $\psi_{F}(t)$ are valid free energies for discrete and continuous spectrum materials and could be included in $(10.1)_{1}$ and indeed (10.2), (10.3). However, it turns out that these converge strongly to $x \psi_{m}(t)+(1-x) \psi_{M}(t)$, where $x \approx 1$, so that $(10.1)$ remains the valid choice for approximating the general form of free energies for continuous spectrum materials, in a manner that preserves the property P4

\section{Free energy functionals for sinusoidal/exponential histories}

Let us explore the work function and various free energy functionals for a history of the strain that is sinusoidal with frequency $\omega_{0}$, combined with exponential behavior giving convergence as $t \rightarrow-\infty$. This combines two simple behaviors studied in [3], for example. Other results on sinusoidal histories may be found in [1] and earlier references.

Consider a history and current value $\left(E^{t}, E(t)\right)$ defined by

$$
E(t)=E_{0} \mathrm{e}^{i \omega_{-} t}+\overline{E_{0}} \mathrm{e}^{-i \omega_{+} t}, \quad E^{t}(s)=E(t-s),
$$

where $E_{0}$ is an amplitude and $\overline{E_{0}}$ its complex conjugate. Furthermore,

$$
\omega_{-}=\omega_{0}-i \eta, \omega_{+}=\bar{\omega}_{-}, \quad \omega_{0}, \eta \in \mathbb{R}^{++}, \quad \omega_{0}=\frac{2 \pi}{t_{\mathrm{p}}},
$$

where $t_{\mathrm{p}}$ is the period introduced in (3.9). The parameter $\eta$ characterizes the exponential factor and ensures finite results. For $\eta \rightarrow 0$, divergences arise in $W(t)$, which render the histories (11.1) unsuitable for the explorations required in the present work. Instead, these will be carried out in Sects. 12 and 13 for histories that are zero for negative times. The purpose of this section is to illustrate, in a particular case, the general conclusions of Sect 3.1.

The quantity $E_{+}^{t}(\omega)$, defined by $(6.10)$, has the form

$$
E_{+}^{t}(\omega)=E_{0} \frac{e^{i \omega_{-} t}}{i\left(\omega+\omega_{-}\right)}+\overline{E_{0}} \frac{\mathrm{e}^{-i \omega_{+} t}}{i\left(\omega-\omega_{+}\right)} .
$$

and the Fourier transform of the relative history $E_{r}^{t}(s)$, namely $E_{r+}^{t}(\omega)$ [see (6.11)], is given by

$$
E_{r+}^{t}(\omega)=E_{+}^{t}(\omega)-\frac{E(t)}{i \omega^{-}}=-E_{0} \frac{\omega_{-}}{\omega^{-}} \frac{\mathrm{e}^{i \omega_{-} t}}{i\left(\omega+\omega_{-}\right)}+\overline{E_{0}} \frac{\omega_{+}}{\omega^{-}} \frac{\mathrm{e}^{-i \omega_{+} t}}{i\left(\omega-\omega_{+}\right)}
$$

From $(3.1)_{2}$ we have

$$
\begin{gathered}
T(t)=\left[G_{0}+G_{+}^{\prime}\left(\omega_{-}\right)\right] E_{0} \mathrm{e}^{i \omega_{-} t}+\left[G_{0}+G_{+}^{\prime}\left(-\omega_{+}\right)\right] \overline{E_{0}} \mathrm{e}^{-i \omega_{+} t} . \\
\text { "161_2016_512_Article" - 2016/6/15 - 9:11 - page 16-\#16 }
\end{gathered}
$$


Equations (11.4) yield that

$$
\begin{aligned}
E_{r+}^{t}\left(-i \alpha_{i}\right) & =-i E_{0} \frac{\omega_{-}}{\alpha_{i}} \frac{\mathrm{e}^{i \omega_{-} t}}{\alpha_{i}+i \omega_{-}}+i \overline{E_{0}} \frac{\omega_{+}}{\alpha_{i}} \frac{\mathrm{e}^{-i \omega_{+} t}}{\alpha_{i}-i \omega_{+}} \\
& =-\frac{e_{i}(t)}{\alpha_{i}}, \quad i=1,2, \ldots, n,
\end{aligned}
$$

in terms of the notation introduced in (9.9).

Any real algebraic quadratic form in $E(t)$ or real functional quadratic form in $E^{t}(s)$ can be written as

$$
V=M E_{0}^{2} \mathrm{e}^{2 i \omega_{0} t}+\bar{M} \bar{E}_{0}^{2} \mathrm{e}^{-2 i \omega_{0} t}+N\left|E_{0}\right|^{2},
$$

in the fully periodic limit, where $\eta \rightarrow 0$. The quantity $N$ must be real. Let us introduce the abbreviated notation

$$
V=\{M, N\} .
$$

Remark 1I.I A quantity $V=\{M, N\}$ will be completely periodic if $M$ and $N$ are finite quantities, independent of $t$.

Consider the quantity

$$
\begin{aligned}
T(t) \dot{E}(t)= & i \omega_{-}\left[G_{0}+G_{+}^{\prime}\left(\omega_{-}\right)\right] E_{0}^{2} \mathrm{e}^{2 i \omega_{-} t} \\
& -i \omega_{+}\left[G_{0}+G_{+}^{\prime}\left(-\omega_{+}\right)\right]{\overline{E_{0}}}^{2} \mathrm{e}^{-2 i \omega_{+} t} \\
& +i\left[\left(\omega_{-}-\omega_{+}\right) G_{0}+\omega_{-} G_{+}^{\prime}\left(-\omega_{+}\right)-\omega_{+} G_{+}^{\prime}\left(\omega_{-}\right)\right]\left|E_{0}\right|^{2} \mathrm{e}^{i\left(\omega_{-}-\omega_{+}\right) t} .
\end{aligned}
$$

In the limit $\eta \rightarrow 0$, this converges to a finite result of the form

$$
T(t) \dot{E}(t)=\left\{i \omega_{0}\left[G_{0}+G_{+}^{\prime}\left(\omega_{0}\right)\right],-2 \omega_{0} G_{s}^{\prime}\left(\omega_{0}\right)\right\}
$$

using the notation of (11.8).

\subsection{The work function}

The work $W(t)$ done on the material to achieve the state $\left(E^{t}, E(t)\right)$ is obtained from $(2.11)$ by integrating the form (11.9). We obtain

$$
\begin{aligned}
W(t)= & \frac{1}{2}\left[\left[G_{0}+G_{+}^{\prime}\left(\omega_{-}\right)\right] E_{0}^{2} \mathrm{e}^{2 i \omega_{-} t}\right. \\
& \left.+\left[G_{0}+G_{+}^{\prime}\left(-\omega_{+}\right)\right] \bar{E}_{0}^{2} \mathrm{e}^{-2 i \omega_{+} t}\right] \\
& +\left[-2 i \eta G_{0}+\omega_{-} G_{+}^{\prime}\left(-\omega_{+}\right)-\omega_{+} G_{+}^{\prime}\left(\omega_{-}\right)\right]\left|E_{0}\right|^{2} \frac{i \mathrm{e}^{2 \eta t}}{2 \eta} .
\end{aligned}
$$

This quantity diverges as $\eta \rightarrow 0$, as noted in the general case after (3.16). We can write an approximate version of this $[1,2]$ in the notation of $(11.8)$ :

$$
\begin{aligned}
W(t) & =\{M, N\}, \\
M & =\frac{1}{2}\left[G_{0}+G_{+}^{\prime}\left(\omega_{0}\right)\right] \\
N & =G_{0}+G_{c}^{\prime}\left(\omega_{0}\right)-\omega_{0} \frac{\partial}{\partial \omega_{0}} G_{c}^{\prime}\left(\omega_{0}\right)-G_{s}^{\prime}\left(\omega_{0}\right) \omega_{0}\left(2 t+\frac{1}{\eta}\right) .
\end{aligned}
$$

We see that in the limit $\eta \rightarrow 0$, the quantity $N$ does not meet the criterion specified in Remark 11.1 , so that $W(t)$ does not obey condition P4. Comparing the singular terms in $N$ with those in $\mathcal{D}_{m}(t)$, the total dissipation for the minimum free energy ([1], page 260), it is clear that they cancel. This is a general property, as noted after (3.16). 
11.2 Free energies

The general form of the free energy given by $(4.1)_{3}$ becomes

$$
\begin{aligned}
\psi(t) & =\{M, N\}, \\
M & =\frac{1}{2}\left[G_{\infty}-\omega_{0}^{2} \widetilde{G}_{+-}\left(\omega_{0},-\omega_{0}\right)\right], \\
N & =\frac{1}{2} \omega_{0}^{2}\left[\widetilde{G}_{+-}\left(\omega_{0}, \omega_{0}\right)+\widetilde{G}_{+-}\left(-\omega_{0},-\omega_{0}\right)\right]=\omega_{0}^{2} \widetilde{G}_{+-}\left(\omega_{0}, \omega_{0}\right),
\end{aligned}
$$

where

$$
\begin{aligned}
\widetilde{G}_{+-}\left(\omega_{1}, \omega_{2}\right) & =\int_{0}^{\infty} \int_{0}^{\infty} \tilde{G}(s, u) \mathrm{e}^{-i \omega_{1} s+i \omega_{2} u} \mathrm{~d} s \mathrm{~d} u \\
& =\overline{\widetilde{G}}_{+-}\left(-\omega_{1},-\omega_{2}\right)=\overline{\widetilde{G}_{+-}}\left(\omega_{2}, \omega_{1}\right) .
\end{aligned}
$$

Special cases of (11.13) include the minimum and related free energies and also the Dill and Fabrizio free energy functionals, all of which are FMSs. It follows from (11.13) that a free energy given by $(4.1)_{3}$ has the property P4 of complete periodicity if $\widetilde{G}_{+-}\left(\omega_{0}, \omega_{0}\right)$ and $\widetilde{G}_{+-}\left(\omega_{0},-\omega_{0}\right)$ are finite. Indeed, explicit relations of the form (11.8) for the $\psi_{f}(t)$, together with the Dill and Fabrizio free energies, were given in [3]. In all cases, $M$ and $N$ were finite, so that these quantities obey P4. Indeed, it is conjectured in [4], on the basis of considerable evidence, that all non-degenerate free energies of the form (4.1) are FMSs. An example of a degenerate form of (4.1) is given by (4.11). Another is the Graffi-Volterra free energy ([1], page 128 and earlier references therein).

\section{Free energy functionals for sinusoidal/exponential histories which vanish for $t<0$}

For discrete spectrum materials, in particular those defined by (8.2), we give expressions for the work function and the free energy functionals $\psi_{f}(t), \psi_{\text {Dill }}(t)$ and $\psi_{F}(t)$, in the case of a history of the strain that vanishes at negative times. Recalling (7.1), we see that (3.8) becomes

$$
\begin{aligned}
& T(t)=T_{\mathrm{e}}(t)+\sum_{i=1}^{n} G_{i} S_{i}(t), \\
& S_{i}(t)=\mathrm{e}^{-\alpha_{i} t} \int_{0}^{t} \mathrm{e}^{\alpha_{i} s} \dot{E}(s) \mathrm{d} s, \quad t \geq 0 .
\end{aligned}
$$

Similarly, (4.8) becomes

$$
\psi(t)=\phi(t)+\frac{1}{2} \sum_{i, j=1}^{n} C_{i j} S_{i}(t) S_{j}(t)
$$

in the light of $(7.10)$

Let us now specialize to the case where the history, at positive times, is sinusoidal with frequency $\omega_{0}$, but combined with exponential behavior. Consider a history and current value $\left(E^{t}, E(t)\right)$ defined by

$$
\begin{aligned}
E(t) & =-i E_{1}\left[\mathrm{e}^{i \omega_{-} t}-\mathrm{e}^{-i \omega_{+} t}\right]=2 E_{1} \mathrm{e}^{\eta t} \sin \left(\omega_{0} t\right), \quad t>0, \\
& =0, \quad t \leq 0, \\
E^{t}(s) & =E(t-s),
\end{aligned}
$$

where the $\omega_{ \pm}$are defined by (11.2) and $2 E_{1} \mathrm{e}^{\eta t}$ is the amplitude. The parameter $\eta$ determines the exponential factor. In the present context, it will be allowed have both positive, negative and zero values. Equation (12.3) yields that

$$
\dot{E}(t)=E_{1}\left[\omega_{-} \mathrm{e}^{i \omega_{-} t}+\omega_{+} \mathrm{e}^{-i \omega_{+} t}\right], \quad t>0 .
$$


${ }_{644}$ Thus,

82

$$
S_{i}(t)=2 E_{1} \operatorname{Re}\left\{\frac{\omega_{-}}{\alpha_{i}+i \omega_{-}}\left[\mathrm{e}^{i \omega_{-} t}-\mathrm{e}^{-\alpha_{i} t}\right]\right\} .
$$

Also, from (2.11), (12.1) and (12.4),

$$
\begin{aligned}
W(t)= & \phi(t)+\sum_{i=1}^{n} \int_{0}^{t} G_{i} S_{i}(u) \dot{E}(u) \mathrm{d} u \\
= & \phi(t)+2 E_{1}^{2} \sum_{i=1}^{n} G_{i} \operatorname{Re}\left\{\left[\frac{\omega_{-}}{2 i\left(\alpha_{i}+i \omega_{-}\right)}\left(\mathrm{e}^{2 i \omega_{-} t}-1\right)\right.\right. \\
& +\frac{\left|\omega_{-}\right|^{2}}{2 \eta\left(\alpha_{i}+i \omega_{-}\right)}\left(\mathrm{e}^{2 \eta t}-1\right) \\
& \left.+\frac{2 \omega_{-}}{\left(\alpha_{i}+i \omega_{-}\right)} \operatorname{Re}\left[\frac{\omega_{-}}{\left(\alpha_{i}-i \omega_{-}\right)}\left(\mathrm{e}^{\left(i \omega_{-}-\alpha_{i}\right) t}-1\right)\right]\right\}
\end{aligned}
$$

The quantity $\psi_{f}(t)$ becomes

$$
\psi_{f}(t)=\phi(t)+H_{\infty} \sum_{i, j=1}^{n} \frac{R_{i}^{f} R_{j}^{f}}{\left(\alpha_{i}+\alpha_{j}\right) \alpha_{i} \alpha_{j}} S_{i}(t) S_{j}(t),
$$

by virtue of (9.12) and (12.2), where $S_{i}(t)$ has the form (12.5).

If $\eta=-|\eta|<0$, the exponential behavior of the history is decaying for large times rather than increasing. The measure of distance (4.9) will be used for histories (12.3), where $\eta<0$, while for $\eta>0,(4.10)$ will be adopted.

It was observed after (10.3) that $\psi_{M}(t)$ rapidly approaches $W(t)$ for increasing exponential histories, as $n$ gets larger. We now show that matters are more complex for decaying exponential histories. This issue is also explored numerically in Sect. 13.

As $t$ increases, we have from (12.6) for $\eta<0$ that

(n) contrast,

$$
\begin{gathered}
W(t) \rightarrow-2 E_{1}^{2} \sum_{i=1}^{n} \int_{0}^{t} G_{i} \operatorname{Re}\left\{\frac{\omega_{-}}{2 i\left(\alpha_{i}+i \omega_{-}\right)}+\frac{\left|\omega_{-}\right|^{2}}{2 \eta\left(\alpha_{i}+i \omega_{-}\right)}\right. \\
\left.+\frac{2 \omega_{-}}{\left(\alpha_{i}+i \omega_{-}\right)} \operatorname{Re}\left[\frac{\omega_{-}}{\left(\alpha_{i}-i \omega_{-}\right)}\right]\right\}=\Lambda \neq 0 .
\end{gathered}
$$

that the approximate equality $\psi_{M}(t) \approx W(t)$ does not hold at large values of $t$

Also, from (9.18),

$$
\psi_{\text {Dill }}(t)=\phi(t)+\frac{1}{2} \sum_{i=1}^{n} G_{i} s_{i}^{2}(t), \quad t \geq 0 .
$$

The functional $\psi_{F}(t)$ is defined by (9.19). Note that, from (9.20),

$$
I^{t}(\tau)=\sum_{i=1}^{n} G_{i} S_{i}(t) \mathrm{e}^{-\alpha_{i} \tau}, \quad t \geq 0,
$$

so that

$$
\begin{aligned}
\psi_{F}(t) & =\phi(t)+\frac{1}{2} \sum_{i, j=1}^{n} C_{i j}^{F} S_{i}(t) S_{j}(t), \quad t \geq 0, \\
C_{i j}^{F} & =\alpha_{i} \alpha_{j} G_{i} G_{j} \int_{0}^{\infty} \frac{\mathrm{e}^{-\left(\alpha_{i}+\alpha_{j}\right) \tau}}{\sum_{l=1}^{n} \alpha_{l} G_{l} \mathrm{e}^{-\alpha_{l} \tau}} .
\end{aligned}
$$


The functionals $\psi_{\text {Dill }}(t)$ and $\psi_{F}(t)$, given by (12.10) and (12.12), were plotted for various parameters and found to be remarkably close to $\psi_{m}(t)$. They were therefore replaced by $\psi_{m}(t)$ and omitted from further explicit consideration, as indicated in Remark 10.3.

\section{Numerical results relevant to the method for approximating continuous spectrum materials}

The proposals put forward in Sect. 10 indicate how the objective set down in Remark 5.3 may be achieved. In this section, we will present numerical results relating to these proposals, based on particular materials defined below and the histories introduced in Sect. 12. These numerical calculations are carried out using MATLAB.

Consider the discrete spectrum approximation to the continuous spectrum theory as given by (8.1) and (8.2), yielding (12.7). This produces, for $f=1$, a minimum free energy which should approximate the continuous spectrum formulae in [10] more and more closely, as we increase the number of sub-divisions of the interval $[a, b]$ in (8.1). This will be checked using (9.16) and turns out to be remarkably accurate, even for low values of $n$.

The discrete spectrum approximation also gives a maximum free energy, as described by Remark 9.1, which was shown in [3] to converge rapidly to $W(t)$, as $n$ increases, at least for diverging exponential histories. Indeed, for $n=4$, the two quantities are virtually equal, at least for the parametric values in that reference. It will turn out that this property does not always hold.

Here, we explore the behavior of the maximum free energy for increasingly large values of $n$ in (8.1). It is established that convergence to a unique functional, not necessarily $W(t)$, occurs, and that $n=8$ is the value at which, for practical purposes, this is achieved Therefore, this approach also yields 126 intermediate free energies $\psi_{f}(t), f=2,3, \ldots, 127$. All the free energies in (10.1) have the property P4.

Finally, we examine the validity of (10.2).

The material parameters and those of the histories are now specified. Dimensions will be removed from all expressions for free energies by dividing by $G_{0} E_{1}^{2}$ [3]. This amounts to taking

$$
G_{0}=E_{1}=1 .
$$

Also, $G_{\infty}$, in the dimensionless version, is equal to $G_{\infty} / G_{0}$ in terms of dimensional quantities, and varies in the range $[0,1]$. It is chosen to be 0.1 . We take $g(\alpha)$ in $(7.11)$ to be a constant quantity $g_{0}$, so that (8.2), for the trapezoidal rule, becomes

$$
G_{i}= \begin{cases}\frac{b-a}{n-1} g_{0}, & i=2.3, \ldots, n-1 \\ \frac{b-a}{2(n-1)} g_{0}, & i=1, n,\end{cases}
$$

where $a$ and $b$ are assigned values 0.5 and 1.5 , respectively. From (7.2), it follows that

$$
g_{0}=\frac{1-G_{\infty}}{b-a},
$$

so that

$$
G_{i}= \begin{cases}\frac{1-G_{\infty}}{n-1}, & i=2,3, \ldots, n-1, \\ \frac{1-G_{\infty}}{2(n-1)}, & i=1, n .\end{cases}
$$

Also, the quantities $\alpha_{i}$ are given by $(8.3)_{1}$.

We take $\omega_{0}=1$ in (11.2) and consider values of $\eta / \omega_{0}$ in the range $[0.1,1.0]$ and $[-0.1,-1.0]$.

Let us first check the validity of (9.16) for these materials. Since $g(\alpha)$ is constant, the quantity $K(\alpha)$, defined by $(9.14)_{2}$, has the form

$$
K(\alpha)=-g_{0}\left[b-a-\alpha \log \left(\frac{b+\alpha}{a+\alpha}\right)\right], \quad \alpha \notin[-b,-a],
$$

while $L(\alpha)$, given by $(9.14)_{3}$, becomes

$$
\begin{gathered}
L(\beta)=-g_{0}\left[b-a+\beta \log \left|\frac{b-\beta}{a-\beta}\right|\right], \quad \beta \in(a, b) . \\
\text { "161_2016_512_Article" - 2016/6/15-9:11 - page 20-\#20 }
\end{gathered}
$$




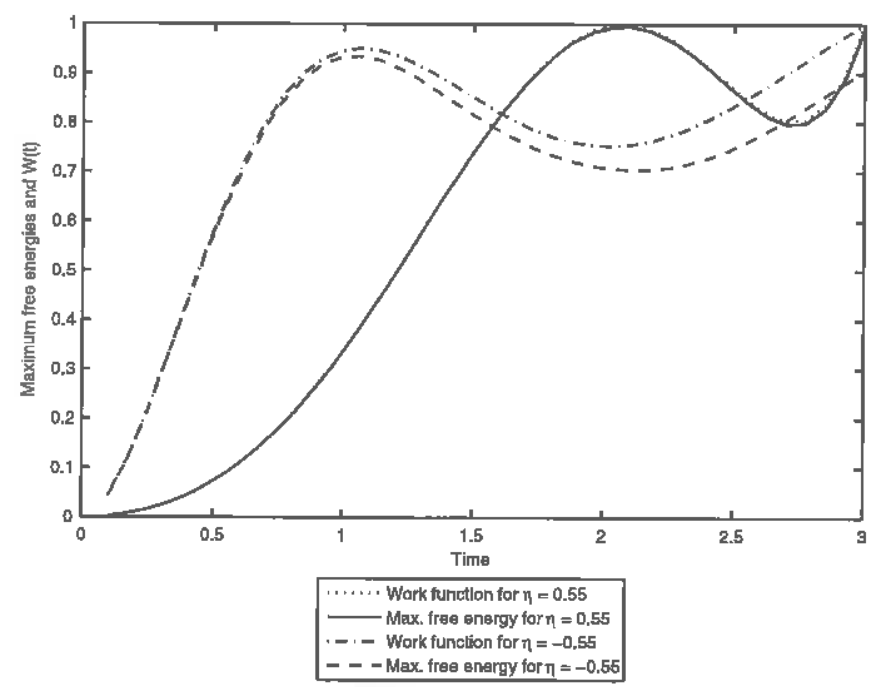

Fig. 1 Maximum free energies for $n=8$ and the work function, for $\eta=0.55$ and -0.55 . These quantities are divided by $W^{(n)}(3)$

We define

$$
C_{i}=\frac{\kappa_{i} \Delta\left(\alpha_{i}\right)}{2 \pi h_{\infty}}, \quad i=1,2, \ldots, n,
$$

where $h_{\infty}$ is given by (9.4) and (7.5). Observe that (9.16) can be written as $C_{i}=R_{i}, i=1,2, \ldots, n$. Values of $C_{i}$ and $R_{i}$, given by (9.7) for $f=1$, corresponding to $\epsilon_{i}^{f}=1, i=2,3, \ldots, n$, were compared for all values of $n$ considered, namely $n=3,4, \ldots 8$. In all cases, the quantities $C_{i}$ and $R_{i}$ were virtually identical.

Recall that (9.16) follows from a comparison of (9.15) (in the light of (7.10)) and (9.12). The numerical results are a good check on these results or on the formulae (9.13), (9.14) on the one hand, and (9.11) for $f=1$, on the other. It should be emphasized that the derivations of these two sets of results are entirely different. The level of agreement between them also provides support for the discretization procedure outlined in Sect. 8 and in (9.15).

The notation $\psi_{f}^{(n)}(t)$ will be used to denote $(12.7)$ and $W^{(n)}(t)$ for $(12.6)$. The superscript $(n)$ on these quantities was omitted up to now to minimize cumbersome notation.

Next, we consider whether $\psi_{M}^{(n)}(t)$ approaches $W^{(n)}(t)$, as $n$ increases. This issue has been addressed earlier by (12.8), (12.9) and after (10.3). The data plotted on Fig. 1 indicates that this is true to a high degree of accuracy for $\eta>0$. Indeed, the convergence is rapid. For $n=8$, they are virtually identical and are very close from $n=4$ onwards.

However, for $\eta<0$, there is rapid convergence on the interval $t \in(0.0,1.0)$ approximately, while for larger times, we have a perceptible difference for $n=8$. This is consistent with the observation made after (12.9).

Thus, in general, $\psi_{M}^{(n)}(t)$ converges to $W^{(n)}(t)$ on a subset $\mathcal{C}$ of $\mathbb{R}^{+}$, where, in some but not all cases, $\mathcal{C}=\mathbb{R}^{+}$.

The next question to explore is whether $\psi_{M}^{(n)}(t)$ converges to a definite value, which may or may not be very close to $W^{(n)}(t)$ for $t \in \mathbb{R}^{+}$. We know that convergence occurs for $t \in \mathcal{C}$ and for $\eta>0$, we have $\mathcal{C}=\mathbb{R}^{+}$. This is confirmed on Fig. 2, which also illustrates that convergence is virtually complete for $n=4$, as noted in the context of Fig. 1.

For $\eta<0$, we see from Fig. 3 that convergence to a definite function $\psi_{M}^{(8)}(t)$ occurs over the range of times shown. However, the convergence is not uniform. It is very rapid up to about $t=1$, where convergence to $W^{(8)}(t)$ is taking place. For larger times, the convergence is more gradual, but is essentially complete for $n=8$. We conclude that there is evidence that $\psi_{M}^{(n)}(t)$ converges to a definite functional, not always equal to $W^{(8)}(t)$.

Figures 1, 2 and 3 provide an exploration of behavior for a range of values of $t$ and for two values of $\eta$. A similar exploration is presented on Table 1 for a fixed value of time and a range of values of $\eta$. These results provide further support for the properties which emerged from the figures. 


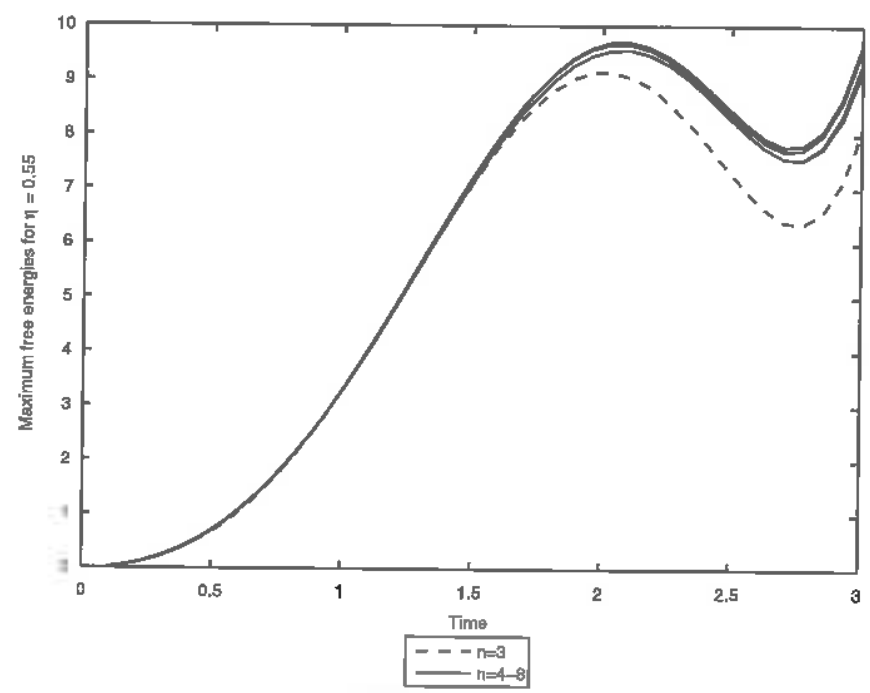

Fig. 2 Maximum free energies for $n=3-8$ and $\eta=0.55$

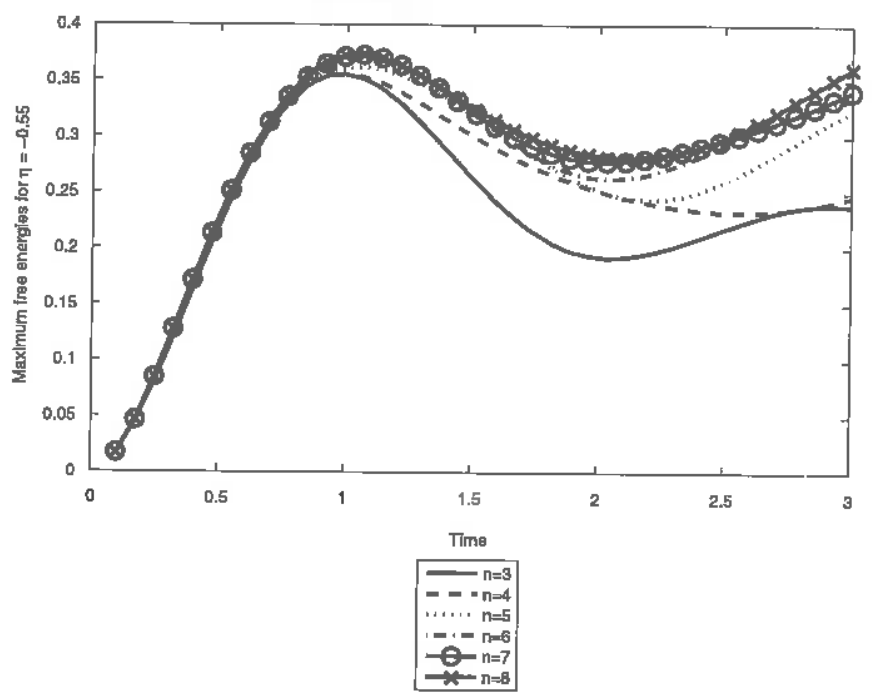

Fig. 3 Maximum free energies for $n=3-8$ and $\eta=-0.55$

Table 1 gives values of $\psi_{m}^{(n)}(t), \psi_{M}^{(n)}(t)$ and

$$
\psi_{a v}^{(n)}(t)=\frac{1}{M-2} \sum_{f=2}^{M-2} \psi_{f}^{(n)}(t)
$$

for $n=3,5,8$ and $t=3.0$. The various quantities are normalized with respect to values of $W^{(n)}(t)$, given by (12.6).

We draw attention to the fact that the minimum free energy values which emerge for different values of $n$ and a specified $\eta$ are in close agreement. This should be the case of course, but, given the quite different formulae that are used to calculate them from (12.7), i.e. the very distinct detailed forms of $R_{i}^{f}$ for $f=1$, increasing in complexity for larger values of $n$, such agreement is supportive of the validity of the approach presented here.

The agreement between $\psi_{M}^{(n)}(t)$ and $W^{(n)}(t)$ where this occurs in the Figs. 1, 2, 3 and Table 1 is also noteworthy, because of the very different algebraic steps taken in the derivation of these two quantities; see

$$
\text { "161_2016_512_Article" — 2016/6/15 - 9:11 — page 22 — \#22 }
$$


Free energies for singleton minimal states

Table 1 The quantities $\psi_{m}^{(n)}(t) / W^{(n)}(t), \psi_{a v}^{(n)}(t) / W^{(n)}(t)$ and $\psi_{M}^{(n)}(t) / W^{(n)}(t)$ for specified values of $n$ and $\eta$ and for $t=3.0$

\begin{tabular}{|c|c|c|c|c|c|c|c|c|c|c|c|}
\hline$n$ below & $\eta=$ & 0.10 & 0.33 & 0.55 & 0.77 & 1.0 & -0.10 & -0.33 & -0.55 & -0.77 & -1.0 \\
\hline \multirow[t]{3}{*}{3} & $\frac{\psi_{M}^{(n)}(t)}{W^{(n)}(t)}$ & 0.82 & 0.84 & 0.84 & 0.82 & 0.80 & 0.77 & 0.7 & 0.61 & 0.51 & 0.43 \\
\hline & $\frac{\psi_{u t}^{(t)}(t)}{W^{(n)}(t)}$ & 0.25 & 0.29 & 0.34 & 0.41 & 0.48 & 0.22 & 0.19 & 0.17 & 0.16 & 0.14 \\
\hline & $\frac{\psi_{m}^{(n)}(t)}{W^{(n)}(t)}$ & 0.22 & 0.22 & 0.22 & 0.19 & 0.16 & 0.2 & 0.17 & 0.14 & 0.11 & 0.09 \\
\hline \multirow[t]{3}{*}{5} & $\frac{\psi_{M}^{(n)}(t)}{W^{(n)}(t)}$ & 0.91 & 0.94 & 0.96 & 0.98 & 0.99 & 0.89 & 0.85 & 0.81 & 0.76 & 0.71 \\
\hline & $\frac{\psi_{a_{v}}^{(n)}(t)}{W^{(n)}(t)}$ & 0.65 & 0.69 & 0.73 & 0.75 & 0.77 & 0.6 & 0.54 & 0.48 & 0.42 & 0.37 \\
\hline & $\frac{\psi_{m}^{m}(t)}{W^{(n)}(t)}$ & 0.22 & 0.23 & 0.22 & 0.2 & 0.16 & 0.2 & 0.17 & 0.14 & 0.11 & 0.09 \\
\hline \multirow[t]{3}{*}{8} & $\frac{\psi_{M}(t)}{W^{(n)}(t)}$ & 0.97 & 0.98 & 0.99 & 1.0 & 1.0 & 0.95 & 0.93 & 0.9 & 0.87 & 0.84 \\
\hline & $\frac{\psi_{a r}^{(n)}(f)}{W^{(n)}(t)}$ & 0.85 & 0.88 & 0.91 & 0.92 & 0.93 & 0.81 & 0.76 & 0.7 & 0.64 & 0.58 \\
\hline & $\frac{\psi_{m}^{(n)}(t)}{W^{(n)}(t)}$ & 0.22 & 0.23 & 0.22 & 02 & 0.17 & 0.2 & 0.17 & 0.14 & 0.11 & 0.09 \\
\hline
\end{tabular}

Table 2 The average values of $x_{\hat{\beta}}^{(n)}$ for specified values of $n$ (rows) and $\eta$ (columns)

\begin{tabular}{|c|c|c|c|c|c|c|c|c|c|c|}
\hline \multirow[t]{2}{*}{$n$} & \multicolumn{10}{|l|}{$\eta$} \\
\hline & 0.10 & 0.33 & 0.55 & 0.77 & 1.0 & -0.10 & -0.33 & -0.55 & -0.77 & -1.0 \\
\hline 3 & 0.51 & 0.51 & 0.49 & 0.45 & 0.4 & 0.56 & 0.57 & 0.58 & 0.48 & 0.53 \\
\hline 4 & 0.35 & 0.32 & 0.31 & 0.31 & 0.31 & 0.4 & 0.42 & 0.47 & 0.43 & 0.4 \\
\hline 5 & 0.26 & 0.23 & 0.21 & 0.2 & 0.2 & 0.33 & 0.35 & 0.38 & 0.42 & 0.43 \\
\hline 6 & 0.2 & 0.17 & 0.15 & 0.14 & 0.14 & 0.24 & 0.28 & 0.3 & 0.32 & 0.35 \\
\hline 7 & 0.15 & 0.12 & 0.11 & 0.1 & 0.09 & 0.19 & 0.23 & 0.27 & 0.3 & 0.33 \\
\hline 8 & 0.12 & 0.09 & 0.07 & 0.06 & 0.06 & 0.16 & 0.19 & 0.23 & 0.27 & 0.3 \\
\hline
\end{tabular}

(12.6) and (12.7). From Table 1, we perceive that this agreement is most marked for large positive values of $\eta$, say from 0.33 upwards.

It might be speculated that the intermediate free energies $\psi_{f}(t)$, and indeed the quantity $\psi_{a v}^{(n)}(t)$ defined by (13.8), merge with $\psi_{M}(t)$ or $\psi_{m}(t)$, as $n$ increases, so that, in the limit, (10.3) holds. This would be a special case of $(10.2)$, where $x \approx 0$ or $x \approx 1$.

We see from Table 1 that $\psi_{a v}^{(n)}(t)$ approaches $W^{(n)}(t)$ (and therefore $\psi_{M}^{(n)}(t)$ ) for positive $\eta$, though the convergence is not particularly strong. For negative $\eta, \psi_{a v}^{(n)}(t)$ remains less than $W^{(n)}(t)$, most notably for large values of $|\eta|$. Thus, (10.3) has approximate validity for positive $\eta$.

This is not a very clear result, so we explore in a more general way the proposal expressed by (10.2), leading to (10.3). Referring to (4.9) and (4.10), we consider the quantity

$$
F_{n}(x)= \begin{cases}\left\|\psi_{f}^{(n)}-x \psi_{m}^{(n)}-(1-x) \psi_{M}^{(n)}\right\|, & \text { bounded histories, tending to zero, } \\ \left\|\left[\psi_{f}^{(n)} / \psi_{M}^{(n)}\right]-x\left[\psi_{m}^{(n)} / \psi_{M}^{(n)}\right]-1+x\right\|, & \text { unbounded histories, }\end{cases}
$$

as a function of $x$. This quantity is varied in the range $(0,1)$ for a given $n$ and $\eta$, yielding the value $x_{0}^{(n)}$ at which $F_{n}(x)$ is minimum. The quantities $x_{0}^{(n)}$ and $F_{n}\left(x_{0}^{(n)}\right)$ are averaged over the parameter $f$. These averages are presented on Tables 2 and 3.

From Table 2, we see that all averages of $x_{0}^{(n)}$ decline with increasing $n$. For positive $\eta$, the values of $x_{0}^{(n)}$ are close to zero, while, for negative $\eta$, they have an average of about 0.23 .

The percentage reduction in the average of $F_{n}\left(x^{(n)}\right)$ as $n$ increases from 3 to 8 is, on average, 88 percent for positive choices of $\eta$, and 69 percent for negative $\eta$. The values of $F_{8}\left(x_{0}^{(8)}\right)$ are small, for all $\eta$, and surprisingly similar, given the different formulae, in the two cases presented by (13.9). Thus, Table 3 yields evidence of a 
Table 3 The average values of $F_{n}\left(x_{0}^{(n)}\right)$ for specified values of $n$ (rows) and $\eta$ (columns)

\begin{tabular}{lllllllllll}
\hline$n$ & $n$ & 7 & 7 & & & & \\
\cline { 2 - 9 } & 0.10 & 0.33 & 0.55 & 0.77 & 1.0 & -0.10 & -0.33 & -0.55 & -0.77 & -1.0 \\
\hline 3 & 0.33 & 0.3 & 0.29 & 0.26 & 0.24 & 0.3 & 0.15 & 0.06 & 0.04 & 0.03 \\
4 & 0.19 & 0.17 & 0.16 & 0.15 & 0.15 & 0.2 & 0.08 & 0.05 & 0.03 & 0.02 \\
5 & 0.13 & 0.11 & 0.1 & 0.09 & 0.09 & 0.14 & 0.08 & 0.04 & 0.03 & 0.02 \\
6 & 0.1 & 0.08 & 0.07 & 0.06 & 0.06 & 0.11 & 0.06 & 0.04 & 0.03 & 0.02 \\
7 & 0.07 & 0.06 & 0.05 & 0.04 & 0.04 & 0.08 & 0.05 & 0.04 & 0.02 & 0.02 \\
8 & 0.05 & 0.04 & 0.03 & 0.03 & 0.03 & 0.07 & 0.04 & 0.03 & 0.02 & 0.02 \\
\hline
\end{tabular}

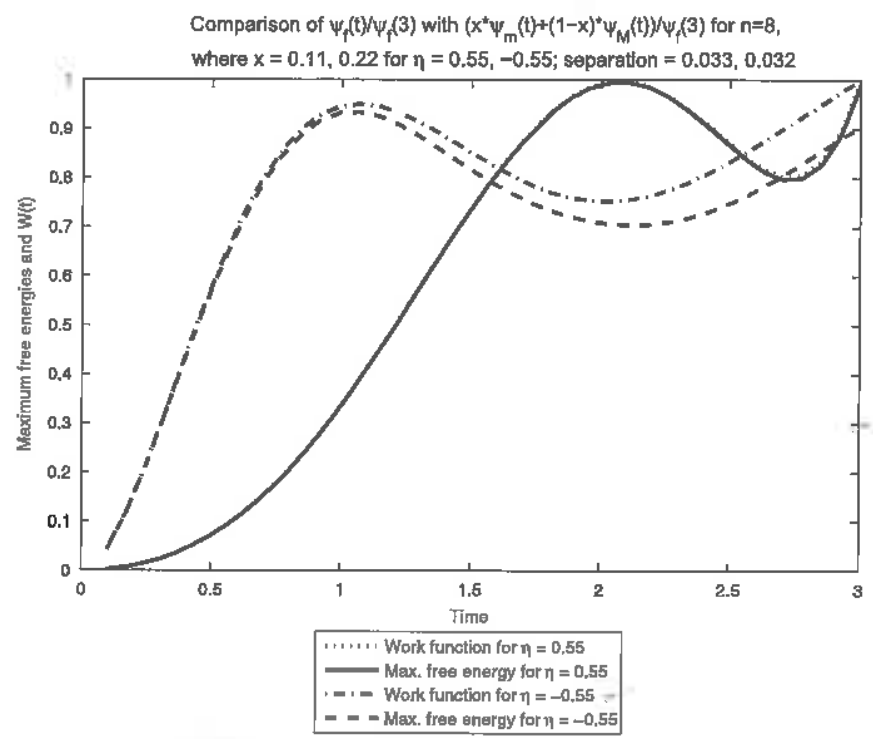

Fig. 4 An example of the difference between $\psi_{f}(t)$ and $x \psi_{m}(t)+(1-x) \psi_{M}(t)$ for $f=93$ if $\eta=0.55$ and $f=99$ if $\eta=-0.55$ These curves are normalized by dividing by $\psi_{f}(3)$, which is equal to 8.45 for $\eta=0.55$ and 0.33 for $\eta=-0.55$. The advantage of normalization is that it allows both sets of curves to be displayed on the same plot

convergence process. There is increasing agreement in (13.9) as $n$ gets larger. For $n=8$, the level of agreement is good, particularly for positive $\eta$, but remains an approximation.

We show an example on Fig. 4. The free energies for $\eta=0.55$ are much larger than those for $\eta=-0.55$; however, after normalization, as described in the caption, the relative magnitudes are reversed. The percentage difference, in each case, is unchanged by the normalization. The difference for both $\eta=0.55$ and 0.55 is 0.03 , as on Table 3. The normalized difference for positive $\eta$ is $0.03 / 8.45=0.0036$, while for negative $\eta$ it is $0.03 / 0.33=0.1$. These two values measure the differences for the normalized quantities shown in Fig. 4 .

The values of $f$ specified in Fig. 4 are chosen from those generated by the algorithm described in Remark 9.2, to achieve separations of around 0.03 in both cases.

Thus (103) will be a reasonably good representation of (10.1).

While there is nothing universally conclusive about these results for a particular material and special histories, it is clear that they support the discretization proposals stated in Sect. 10 and the replacement of (5.3) by (10.1). The further step of replacing (10.1) by (10.3) is approximately valid and is justified by the much greater simplicity of (10.3).

Acknowledgments This research was supported by the Dublin Institure of Technology.

Compliance with ethical standards

Conflict of interest The author declares that he has no conflict of interest.

$$
\text { "161_2016_512_Article" — 2016/6/15 — 9:11 — page 24 — \#24 }
$$




\section{References}

1. Amendola, G., Fabrizio, M., Golden, J.M.: Thermodynamics of Materials with Memory: Theory and Applications. Springer, New York (2012)

2. Amendola, G., Fabrizio, M., Golden, J.M.: Free energies in a general non-local theory of a material with memory. Math. Models Methods Appl. Sci. 24, 1037-1090 (2014)

3. Amendola, G., Fabrizio, M., Golden, J.M.: Algebraic and numerical explotation of free energies for materials with memory. Electron. J. Differ. Equ. 2015, 1-42 (2015)

4. Amendola, G., Fabrizio, M., Golden, J.M.: Free energies and minimal states for scalar linear viscoelasticity. J. Elast. (2016). doi: $10.1007 / \mathrm{s} 10659-015-9549-y$

5. Coleman, B.D.: Thermodynamics of materials with memory Arch. Ration. Mech. Anal. 17, 1-45 (1964)

6. Del Piero, G., Deseri, L.: On the analytic expression of the free energy in linear viscoelasticity. J. Elast. 43, $247-278$ (1996)

7. Del Piero, G., Deseri, L.: On the concepts of state and free energy in linear viscoelasticity. Arch. Ration. Mech. Anal, 138, $1-35(1997)$

8. Deseri, L., Gentili, G., Golden, J.M.: An explicit formula for the munimum free energy in linear viscoelasticity. J. Elast. 54, $141-185$ (1999)

9. Deseri, L.. Fabrizio. M., Golden, J.M.: On the concept of a minimal state in viscoelasticity: new free energies and applications to PDE S. Arch. Ration. Mech. Anal. 181, 43-96 (2006)

10. Deseri, L., Golden, J.M.: The minimum free energy for continuous spectrum materials. SIAM J. Appl. Math. 67, 869-892 (2007)

11. Fabrizio, M., Morro, A.: Mathematical Problems in Linear Viscoelasticity. SIAM, Philadelphia (1992)

12. Fabrizio, M., Golden, J.M.: Maximum and minimum free energies for a linear viscoelastic material. Q. Appl. Math. 60, 341-381 (2002)

13. Fabrizio, M., Gentili, G., Golden, J.M.: Free energies for linear non-isothermal materials with memory. Math. Comput. Model. 39, 219-253 (2004)

14. Golden, J.M.: Free energies in the frequency domain: the scalar case. Q. Appl. Math. 58, 127-150 (2000)

15. Golden, J.M.: A proposal concerning the physical rate of dissipation in materials with memory. Q. Appl, Math. 63, 117-155 (2005)

16. Golden, J.M.: A proposal concerning the physical dissipation of materials with memory: the non-isothermal case. Math Mech. Solids 12, 403-449 (2007)

17. Golden, J.M.: Constructing free energies for materials with memory. Evol. Equ. Control Theory 3, 447-483 (2014)

18. Golden, J.M.: Free energies for materials with memory in terms of state functionals. Meccanica 49, 2207-2235 (2014)

19. Golden, J.M.: The minimum and other free energies for non-linear materials with memory. Q. Appl. Math. 74, 137-164 (2016)

20. Golden, J.M.: Unique characterization of materials with memory. Q. Appl, Math. 74, 361-374 (2016)

21. Graff, D.: Sull'expressione analitica di alcune grandezze termodinamiche nei materiali con memoria. Rend. Sem. Mat. Univ Padova 68, 17-29 (1982)

22. Graffi, D., Fabrizio, M:: Sulla nozione di stato materiali viscoelastici di tipo 'rate'. Atti Accad. Naz. Lincei 83, 201-208 (1990)

23. Graffi, D.: Ancora sull'expressione analitica dell'energia libera nei materiali con memoria. Atti Acc. Sci. Torino 120, 111-124 (1986)

24. Noll, W.: A new mathematical theory of simple materials. Arch. Ration. Mech. Anal. 48, 1-50 (1972) 